\title{
Photoionization and electron-ion recombination: The carbon sequence
}

\author{
Sultana N. Nahar and Anil K. Pradhan \\ Department of Astronomy, Ohio State University, Columbus, Ohio 43210
}

(Received 12 April 1991)

\begin{abstract}
We describe close-coupling calculations for photoionization and recombination using the $R$-matrix method. Photoionization of a large number of bound states of each atom or ion is considered, and cross sections are computed at a fine mesh of energies to delineate the extensive resonance structures. Photoionization of ground and excited configurations with $n \leq 10$ and $l \leq 3$ is considered. Partial photoionization cross sections into excited states of the residual ion are also obtained for the ground states of $\mathrm{C}^{0}, \mathrm{~N}^{+}$, and $\mathrm{O}^{2+}$. An important type of autoionizing resonances due to the photoexcitation of the core ion is discussed. As the photoionization cross sections incorporate autoionization in detail, it is possible in principle to employ the Milne relation to obtain total, radiative plus dielectronic, recombination cross sections and rate coefficients. However, practical difficulties arise, and various approaches are described to overcome these. Comparison is made with the earlier results for photoionization cross sections and recombination coefficients. This work forms an extension of the Opacity Project.
\end{abstract}

\section{INTRODUCTION}

The Opacity Project [1-3] is an extensive effort to compute huge quantities of accurate atomic data for radiative processes of importance in the calculation of astrophysical opacities. An $a b$ initio quantum-mechanical formulation is employed, based on the close-coupling approximation and the $R$-matrix method. For the Opacity Project work, as well as for extensions to collisional processes, computer programs have been developed to facilitate the large-scale calculations for electron-ion-scattering cross sections, photoionization cross sections, radiativetransition probabilities, line damping constants, etc. Calculations are carried out along isoelectronic sequences beginning with elaborate configuration-interaction (CI) expansion for the target or the "core" ions. The computational methods are capable of rapid calculation of boundand continuum-state coupled-channel wave functions that are employed to obtain the relevant matrix elements to yield the atomic parameters.

Prior to the Opacity Project, relatively few accurate photoionization cross sections could be computed and have been mainly done for the ground state of the given atom or ion. It is now possible to consider the photoionization of a very large number of bound states involving high degrees of excitation. For the Opacity Project a practical limit was set at excited states with an effective quantum number $v \leq 10.0$ and the orbital quantum number $l \leq 3$ and 4 or 5 for the second row and the fourth row elements of the Periodic Table. The calculation extends from hydrogen up to iron, in all ionization stages.

In previous papers we have reported on the oscillator strengths for the carbon isoelectronic sequence [4] and electron-ion-scattering cross sections [5] for the boronlike ions using the same eigenstate expansion. In the present paper we focus on the photoionization calculations in detail and describe an important extension of the work: the total (electron plus ion) recombination problem. The photoionization calculations reported herein represent slight improvements (described later) over the earlier Opacity Project results for the carbon sequence.

It is the usual practice to consider electron plus ion recombination in separate parts: radiative and dielectronic. Radiative recombination is regarded as the inverse of the photoionization process without autoionization and dielectronic recombination is regarded as the inverse of autoionization followed by radiative decay. However, since the photoionization cross sections described in this work include autoionizing resonances in an $a b$ initio manner, the principle of detailed balance may be employed to obtain the cross section for total effective $e$ plus ion recombinations, without invoking the essentially artificial distinction between the two recombination processes. Furthermore, the interference between the two modes of recombination is taken into account, whereas a separate treatment could involve serious errors, for example, in the case of overlapping resonances. The detailed balance equivalence between photoionization and recombination cross sections is expressed by the Milne relation [6], which yields

$$
\sigma_{\mathrm{RC}}=\frac{\omega_{i}}{\omega_{i+1}} \frac{h^{2} v^{2}}{m^{2} c^{2} v^{2}} \sigma_{\mathrm{PI}}
$$

where $\omega_{i}$ and $\omega_{i+1}$ are the statistical weight factors of the recombined and recombining ions, respectively. Given a velocity distribution $f(v, T)$ at temperature $T$, one then obtains the recombination-rate coefficient

$$
\alpha_{R}(T)=\int v f(v, T) \sigma_{\mathrm{RC}}(v) d v,
$$

where $\sigma_{\mathrm{RC}}$ can be written in terms of $\sigma_{\mathrm{PI}}$ using Eq. (1). If $f(v, T)$ is taken to be the Maxwellian, then in the context of the recombination problem the negative exponential factor $\exp \left[-m v^{2} /(2 k T)\right]$ implies these points: (i) the structure in $\sigma_{\mathrm{PI}}$ may be important, as when autoionizing 
resonances are present, and the detailed profiles of the features need to be determined precisely; (ii) resonance features lying close to the ionization threshold could make a large contribution and therefore the positions of the resonances should be known accurately; and (iii) all resonances should be accounted for; therefore the number of features in $\sigma_{\mathrm{PI}}$ should correspond to the number of autoionizing states expected to be present (i.e., this needs to be verified independently).

Thus, provided the profiles, position, and the number of resonances are determined precisely, the present approach in principle should yield very accurate total recombination cross sections at least in the low-energy region encompassed by the autoionizing resonances explicitly considered in the calculations, i.e., the energy region with $n \leq 10$ below the threshold of convergence for the numerous Rydberg series, corresponding to the coupled channels included in the eigenfunction expansion. In practice, however, there are a number of problems that arise due to finite resolution of resonance structures, particularly for narrow resonances lying close to the ionization threshold. The problems are discussed in detail, along with the approximations employed to overcome them.

For the high-energy region, close to the series limits of the autoionizing resonances with $n>10$, dielectronic recombination would be the dominant contributor to the total recombination. While a simple approximation, such as the Burgess formula [7], may be employed in this region, we describe a more accurate approach based on our previous work and the Bell and Seaton theory of dielectronic recombination $[8,9]$. However, the emphasis of this work is the "low"-energy region corresponding to the photoionization of the bound states of the ( $e$ plus ion) system. Comparisons are made with earlier low- $n$ dielectronic recombination calculations of Nussbaumer and Storey [10].

\section{THE R-MATRIX METHOD FOR BOUND-FREE CROSS SECTIONS}

The second paper in the series of publications Atomic Data For Opacity Calculations (ADOC II) [3] describes the extension of the original $R$-matrix method [11] for the Opacity Project.

\section{A. Wave-function expangions}

We describe the ion as an $N$-electron "target" (the term is borrowed from collisional work) or the "core," with wave functions for the core states represented by

$$
\chi\left(\Gamma_{i} S_{i} L_{i} M_{S_{i}} M_{L_{i}} \pi_{i} \mid x\right)
$$

for each state $S_{i} L_{i} \pi_{i}$ of the ion ( $x$ denotes all coordinates of the core ion). The bound states of the $(N+1)$-electron system then may be expanded in terms of the eigenstates $\chi_{i}$ and the radial part $F_{i}\left(r_{n}\right)$ of the additional electron wave function as

$$
\Psi^{S L \pi}(E)=A^{\prime} \sum_{i=1}^{J} \chi_{i}\left(r_{n}^{-1}\right) \frac{1}{r_{n}} F_{i}\left(r_{n}\right)+\sum_{j=1}^{J} c_{j} \Phi_{j},
$$

where $r_{n}$ denotes the coordinate of the electron and $r_{n}^{-1}$ denotes all coordinates except $r_{n} ; A^{\prime}$ is the antisymmetrization operator for the product of the $\chi_{i}$ and $F_{i}$. The $\Phi_{j}$ in the second sum of the right-hand side of $\mathrm{Eq}$. (1) are $(N+1)$-electron bound system correlation-type functions included to satisfy orthogonality constraints on $F_{i}$ (which must be orthogonal to the core orbitals $P_{n i}$ ) and shortrange correlation effects. $I$ is the number of "free" channels corresponding to the core states included and $J$ is the number of "bound" channels in the close-coupling (CC) expansion for the ( $e$ plus ion) wave function $\Psi$ for a given symmetry $S L \pi$ at total energy $E$. The total $S L \pi$ is defined as the vector sum of the electron and the ion spin and angular momenta and the total parity $\pi=(-1)^{8}$, where $\mathcal{S}=\sum_{i} l_{l}$, i.e., $S L \pi:\left(S_{i} L_{i}\right) l_{i} s_{i}$ where the electron orbital angular momentum is designated as the partial wave $l$. Depending on the number of partial waves in. cluded there may be a large number of total $S L \pi$ states to be considered in the entire calculation.

The $(N+1)$-electron system may be treated with the electron in a bound $(E<0)$ or a continuum $(E>0)$ state, respectively, for the total system. The wave functions $\Psi(S L \pi ; E)$ are expanded in terms of the $R$-matrix basis set of continuum type orbitals within the so-called inner region $r<a$, where $a$ is the $R$-matrix boundary, and asymptotic solutions in the outer region $r>a$; the two solutions are matched at $r=a$. Once the $R$ matrix is obtained through the diagonalization of the $(N+1)$ electron Hamiltonian for each $S L \pi$, the $\Psi(S L \pi ; E)$ may be obtained for all energies; only the asymptotic solutions need to be calculated at each energy.

\section{B. Computational progress}

The coupled integrodifferential (ID) equations of the $\mathrm{CC}$ approximation are formulated using the Kohn variational principle. It is assumed that the core or the target ion wave functions $\chi_{i}$ are known; the $F_{i}(r)$ and the $c_{j}$ (coefficients of the bound channel functions $\Phi_{j}$ ) are treated as the variational parameters that are obtained on solving the coupled ID equations, by diagonalization of the $(N+1)$-electron Hamiltonian through the $R$-matrix method. It is therefore essential to obtain the $\chi_{i}$ as accurately as possible, for input into the ID equations, since any error in $\chi_{i}$ will be propagated through as a first-order error.

Considerable effort is usually devoted in generating the core-ion wave functions using a CI-type expansion. The computer codes employed in this initial step are CIV3 [12] or SUPERSTRUCTURE [13]. The one-electron orbital basis set $\left\{P_{n l}\right\}$ thus obtained can then yield the $\chi_{i}$ through the usual Slater determinant expansions. The $\left\{P_{n l}\right\}$ are input to the $R$-matrix codes consisting of three programs: STG1, STG2, and STGH, which compute the radial integrals, the algebraic coefficients, and carry out the matrix diagonalization, respectively. The output of STGH consists of the energy-independent $R$-matrix basis set of functions and the parameters needed to calculate the dipole matrix elements in the inner region $r<a$.

A subsidiary set of codes is employed to use the $R$ matrix data in order to calculate cross sections, transition 
probabilities, etc. Code STGB is used to obtain the energy levels and radial wave functions for the bound states of ( $e$ plus ion) systems; code STGF is used to compute the continuum-state wave functions including the asymptotic solutions; code STGBB is used to calculate dipole transition probabilities; and code STGBF is for the bound-free (photoionization) cross sections.

\section{Energy mesh}

In order to resolve the extensive autoionization structures, many of which are narrow or overlapping, it is necessary to make a judicious choice of mesh intervals and sizes. Essentially there are two kinds of meshes employed: (i) constant energy interval and (ii) constant interval in effective quantum number.

For most of the calculations a constant energy mesh is unsuitable since the resonances become narrower progressively along the corresponding Rydberg series and a fixed interval in energy would either miss the higher members or leave them underresolved. On the other hand, we may define the effective quantum number $v$ as a variable function of $E$ as

$$
v(E)=\frac{z}{\left[E-E\left(S_{i} L_{i}\right)\right]^{1 / 2}},
$$

relative to the threshold energy $E\left(S_{i} L_{i}\right)$ of the state $S_{i} L_{i}$ of the core ion. The pattern of a Rydberg series of resonances repeats itself when $v$ changes by unity. Thus a constant interval in the $v$ mesh may be specified by $\Delta v$ such that there is a fixed number of $1 / \Delta v$ points for each interval $\Delta v=1$. For example, if $\Delta v=0.01$, then each resonance pattern in the series is resolved by 100 points; this value of $\Delta v=0.01$ is the usual choice. However, a maximum value of $v, v_{\max }$, needs to be specified to terminate the fine tabulation when resonances are so narrow that it is not too meaningful to resolve; $v_{\max }=10.0$ is also the usual choice.

The $E$ mesh is also employed in certain regions, particularly just above the first ionization threshold in order to "search" for missing resonances that might be extremely narrow but important for $e$ plus ion recombination. We shall describe the $E$ mesh later in discussing the recombination calculations.

The resonances occur in the energy range from the first ionization threshold, corresponding to the ground state of the residual ion, up to the highest ion state included in the CC expansion. Above the highest core-ion threshold, all channels are open and the $E$ mesh with a fixed and large $\Delta E$ may be employed.

\section{COMPUTATIONS AND RESULTS FOR PHOTOIONIZATION}

\section{A. Core-ion wave functions}

The optimization of the CI-type wave function representation for the core states of the residual ion is described in detail by Luo and Pradhan [4,5] for the core ions of the boron isoelectronic sequence, in particular $\mathrm{C}^{+}, \mathrm{N}^{2+}$, and $\mathrm{O}^{3+}$. The wave functions for the core states $\chi_{i}$ so obtained are employed in the present calculations. The calculations reported here differ from the previous Opacity Project work in two respects: (i) a much finer resolution for autoionizing resonances likely to be important for recombination and (ii) use of "experimental," rather than the calculated eigenenergies for the core states $\chi_{i}$ so that the energy scale is somewhat more accurate (the differences between the calculated and the experimental core energies are small). Table I shows the states of the core ions $\mathrm{C}^{+}, \mathrm{N}^{2+}$, and $\mathrm{O}^{3+}$ that are included in the CC calculations. Preceded by the number of states included, the calculations may be designated as $10 \mathrm{CC}$ for $\mathrm{C}^{0}$ and $8 \mathrm{CC}$ for $\mathrm{N}^{+}$and $\mathrm{O}^{2+}$.

\section{B. Bound states of the $e$ plus ion system}

We consider all bound states of carbonlike systems $\mathrm{C}^{0}, \mathrm{~N}^{+}$, and $\mathrm{O}^{2+}$ beginning with the $n=2$ ground complex of states dominated by $2 s^{2} 2 p^{2}\left({ }^{3} P,{ }^{1} D,{ }^{1} S\right)$ and $2 s 2 p^{3}\left({ }^{5} S^{0},{ }^{3} D^{0},{ }^{3} S^{0},{ }^{1} D^{0},{ }^{1} S^{\circ},{ }^{1} P^{0}\right)$ up to and including all bound states of the type $2 s^{2} 2 p n l, 2 s 2 p^{2} n l$, $2 s^{2} 3 s n l, 2 s^{2} 3 p n l, 2 p^{3} n l$ with $n \leq 10$ and $l \leq 3$, where $n, l$

TABLE I. Energy levels $E_{c}$ in Rydberg of the target ions.

\begin{tabular}{|c|c|c|c|c|c|}
\hline \multicolumn{2}{|c|}{$\mathrm{C}^{+}$} & \multicolumn{2}{|c|}{$\mathbf{N}^{2+}$} & \multicolumn{2}{|c|}{$\mathrm{O}^{3+}$} \\
\hline State & $E_{c}$ & State & $E_{c}$ & State & $E_{c}$ \\
\hline $2 s^{2} 2 p^{2} P^{\circ}$ & 0.0 & $2 s^{2} 2 p^{2} p^{0}$ & 0.0 & $2 s^{2} 2 p^{2} p^{\circ}$ & 0.0 \\
\hline $2 s 2 p^{24} S$ & 0.3918 & $2 s 2 p^{24} P$ & 0.5209 & $2 s 2 p^{24} P$ & 0.6482 \\
\hline $2 s 2 p^{22} D$ & 0.6824 & $2 s 2 p^{22} D$ & 0.9196 & $2 s 2 p^{22} D$ & 1.1545 \\
\hline $2 s 2 p^{22} S$ & 0.8789 & $2 s 2 p^{22} S$ & 1.1927 & $2 s 2 p^{22} S$ & 1.4955 \\
\hline $2 s 2 p^{22} P$ & 1.0080 & $2 s 2 p^{22} P$ & 1.3289 & $2 s 2 p^{22} P$ & 1.6438 \\
\hline $2 s^{2} 3 s^{2} S$ & 1.0616 & $2 p^{34} S^{0}$ & 1.7012 & $2 p^{34} S^{0}$ & 2.1052 \\
\hline $2 s^{2} 3 p^{2} P^{o}$ & 1.200 & $2 p^{32} D^{\circ}$ & 1.8505 & $2 p^{32} D^{\circ}$ & 2.3230 \\
\hline $2 p^{34} S^{0}$ & 1.2939 & $2 p^{32} P^{0}$ & 2.0986 & $2 p^{32} P^{0}$ & 2.6315 \\
\hline $2 p^{32} D^{\circ}$ & 1.3708 & & & & \\
\hline $2 p^{32} P^{\circ}$ & 1.5373 & & & & \\
\hline
\end{tabular}


refer to the outer electron. The $2 s^{2} 3 s$ and $2 s^{2} 3 p$ configurations are not included for $\mathrm{N}^{+}$and $\mathrm{O}^{2+}$. The eigenenergies and the eigenfunctions are obtained from the program STGB. Spectroscopic identification of the bound states is a nontrivial task that requires a detailed examination of various calculated parameters such as the effective quantum numbers and the fraction of the total wave functions in each of the coupled channels in the asymptotic region $r>a$.

A large number of bound states of $\mathrm{C}^{0}, \mathrm{~N}^{+}$, and $\mathrm{O}^{2+}$ are thus explicitly considered. In Table II we present the ionization energies of the bound states with $n \leq 10$ and $l \leq 2$ (the $l>2$ states are not given since the energies are nearly hydrogenic). A comparison of the computed eigenenergies with available experimental data shows that the present values agree with experiment generally to within $1 \%$, often to $0.1 \%$.

\section{Continuum states of the $e$ plus ion system}

With the combination of $l \leq 4$ and the states $S_{i} L_{i}$ of the core ion as in Table $I$, the total $S L \pi$ states that need to be considered are ${ }^{1,3}(S, P, D, F, G, H, I)^{e}$; ${ }^{1,3}(S, P, D, F, G, H, I)^{\circ} ; \quad{ }^{5}(S, P, D, F, G)^{e} ; \quad{ }^{5}(S, P, D, F, G)^{e}$, a total of $37 S L \pi$ states (" $e$ " denotes even parity and "o" the odd parity). However, a few more $S L \pi$ 's are also included, since we calculate the bound states of all the symmetries listed above, but the photoionization of the highest $L$ states requires the continuum-state wave functions of $\Delta L=0, \pm 1$ according to the dipole selection rules. Therefore, for example, the photoionization of ${ }^{5} H^{o}$ states requires that we also consider ${ }^{5} H^{e}$ and ${ }^{5} I^{e}$.

The continuum-state wave functions (also called the "free" wave functions) are calculated by the program STGF. The free-state wave functions must be obtained at each energy in the energy mesh as mentioned earlier. The energy region from the first ionization threshold up to the highest threshold included in the CC expansion consists of many series of resonances converging up to the excited states of the residual ion listed in Table I. For the most part, a quantum-defect-type energy mesh is employed. However, the tabulation is carried out up to $v_{\max }=10.0$, relative to each residual ion threshold, since the resonances are very narrow in the small energy region $10<v<\infty$. A Gailitis-type resonance averaging procedure [14] is employed to bridge this small gap below each threshold. With $\Delta v=0.01$, we obtain 100 energy points in each interval $(v, v+1)$ and thus it turns out that the energy range encompassing the residual ion thresholds is described by a fine mesh of well over 2000 points for the photoionization of most of the bound states listed in Table II. However, as we shall see later, this mesh still is not fine enough to delineate, or even detect, some of the low-lying narrow resonances that may still be of importance in the recombination process.

\section{Photoionization calculations}

Photoionization cross sections are calculated for all of the bound states listed in Table II. Before the present work was carried out photoionization cross sections were available only for the ground states and a few low-lying metastable states.

As mentioned earlier the present calculations are different from the earlier calculations for the Opacity Project as it was discovered that the calculations of recombination-rate coefficients required a different, finer tabulation. Section II D 1 describes some of the results in detail.

\section{Total and partial cross sections for the ground states}

Total cross sections are calculated for the photoionization of the ground states, as well as the partial contributions to it for photoionization into the individual excited states of the residual ion. All ionization thresholds included in the CC expansion are considered. Cross sections for photoionization into the excited thresholds are often important in the determination of population rates for the corresponding states required, for example, in nonlocal thermodynamic equilibrium astrophysical models.

Figures 1-3 present the total and partial cross sections for the ground state $2 s^{2} 2 p^{2}\left({ }^{3} P\right)$ of $\mathrm{C}^{0}, \mathrm{~N}^{+}$, and $\mathrm{O}^{2+}$. In

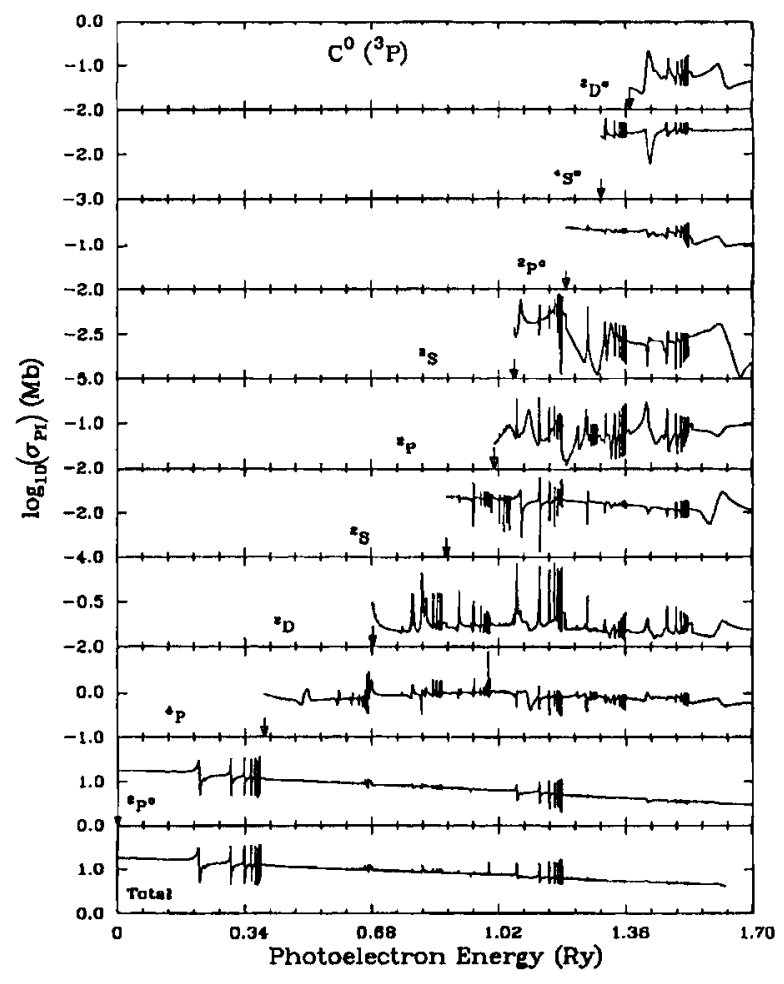

FIG. 1. Photoionization cross sections $\sigma_{\mathrm{PI}}$ (in $\mathrm{Mb}$ ) for the ground state $2 s^{2} 2 p^{23} P$ of $C^{6}$. The lowest panel shows the total cross section and the successively higher ones correspond to partial cross sections from the ground state leaving the residual ion in the states $2 s^{2} 2 p\left({ }^{2} P^{o}\right), 2 s 2 p^{2}\left({ }^{4} P,{ }^{2} D,{ }^{2} S,{ }^{2} P\right), 2 s^{2} 3 s\left({ }^{2} S\right)$, $2 s^{2} 3 p\left({ }^{2} P^{\circ}\right)$, and $2 p^{3}\left({ }^{4} S^{0},{ }^{2} D^{\circ}\right)$. The lower $y$-axis limit in each panel corresponds to the partial cross sections indicated, but the upper limit is not shown except for the top one. 
TABLE II. Calculated ground- and excited-state energies $E(\mathrm{Ry})$ of $\mathrm{C}^{0}, \mathrm{~N}^{+}$, and $\mathrm{O}^{2+}$. Notation $a[b]$ means $a \times 10^{b}$.

\begin{tabular}{|c|c|c|c|c|c|}
\hline State & $\boldsymbol{E}$ & State & $\boldsymbol{E}$ & State & $E$ \\
\hline \multicolumn{6}{|c|}{$\mathbf{C}^{\mathbf{0}}$} \\
\hline $2 s^{2} 2 p^{23} P$ & $-8.452[-1]$ & $2 s^{2} 2 p\left({ }^{2} P^{\circ}\right) 5 d^{1} F^{\circ}$ & $-4.054[-2]$ & $2 s^{2} 2 p\left({ }^{2} P^{o}\right) 8 p^{1} D$ & $-1.823[-2]$ \\
\hline $2 s^{2} 2 p^{21} D$ & $-7.495[-1]$ & $2 s^{2} 2 p\left({ }^{2} P^{\circ}\right) 5 f^{1} F$ & $-4.018[-2]$ & $2 s^{2} 2 p\left({ }^{2} P^{0}\right) 8 p^{1} S$ & $-1.776[-2]$ \\
\hline $2 s^{2} 2 p^{21} S$ & $-6.354[-1]$ & $2 s^{2} 2 p\left({ }^{2} P^{o}\right) 5 f^{3} F$ & $-4.018[-2]$ & $2 s^{2} 2 p\left({ }^{2} P^{o}\right) 8 d^{1} D^{o}$ & $-1.648[-2]$ \\
\hline $2 s 2 p^{3} s^{0}$ & $-5.489[-1]$ & $2 s^{2} 2 p\left({ }^{2} P^{0}\right) 5 f^{3} G$ & $-4.002[-2]$ & $2 s^{2} 2 p\left({ }^{2} P^{o}\right) 8 d^{3} F^{o}$ & $-1.603[-2]$ \\
\hline $2 s^{2} 2 p\left({ }^{2} P^{\circ}\right) 3 s^{3} P^{0}$ & $-2.931[-1]$ & $2 s^{2} 2 p\left({ }^{2} P^{\circ}\right) 5 d^{1} P^{\circ}$ & $-4.002[-2]$ & $2 s^{2} 2 p\left({ }^{2} P^{\circ}\right) 9 s^{3} P^{o}$ & $-1.593[-3]$ \\
\hline $2 s^{2} 2 p\left({ }^{2} P^{o}\right) 3 s^{1} P^{o}$ & $-2.767[-1]$ & $2 s^{2} 2 p\left({ }^{2} P^{o}\right) 5 f^{1} G$ & $-4.002[-2]$ & $2 s^{2} 2 p\left({ }^{2} P^{o}\right) 8 d^{3} D^{\circ}$ & $-1.588[-2]$ \\
\hline $2 s 2 p^{33} D^{\circ}$ & $-2.625[-1]$ & $2 s^{2} 2 p\left({ }^{2} P^{\circ}\right) 5 f^{3} D$ & $-3.996[-2]$ & $2 s^{2} 2 p\left({ }^{2} P^{0}\right) 9 s^{1} P^{o}$ & $-1.584[-2]$ \\
\hline $2 s^{2} 2 p\left({ }^{2} P^{0}\right) 3 p p^{1} P$ & $-2.104[-1]$ & $2 s^{2} 2 p\left({ }^{2} P^{\circ}\right) 5 f^{1} D$ & $-3.996[-2]$ & $2 s^{2} 2 p\left({ }^{2} P^{0}\right) 8 d^{1} F^{0}$ & $-1.576[-2]$ \\
\hline $2 s^{2} 2 p\left({ }^{2} P^{0}\right) 3 p^{3} D$ & $-2.015[-1]$ & $2 s^{2} 2 p\left({ }^{2} P^{\circ}\right) 5 d^{3} P^{\circ}$ & $-3.994[-2]$ & $2 s^{2} 2 p\left({ }^{2} P^{o}\right) 8 f^{1} F$ & $-1.568[-2]$ \\
\hline $2 s^{2} 2 p\left({ }^{2} P^{o}\right) 3 p{ }^{3} S$ & $-1.904[-1]$ & $2 s^{2} 2 p\left({ }^{2} P^{\circ}\right) 6 p{ }^{1} P$ & $-3.663[-2]$ & $2 s^{2} 2 p\left({ }^{2} P^{o}\right) 8 f^{3} F$ & $-1.568[-2]$ \\
\hline $2 s^{2} 2 p\left({ }^{2} P^{o}\right) 3 p^{3} P$ & $-1.825[-1]$ & $2 s^{2} 2 p\left({ }^{2} P^{0}\right) 6 p^{3} D$ & $-3.624[-2]$ & $2 s^{2} 2 p\left({ }^{2} P^{0}\right) 8 d^{3} P^{\circ}$ & $-1.564[-2]$ \\
\hline $2 s^{2} 2 p\left(^{2} P^{o}\right) 3 p^{1} D$ & $-1.686[-1]$ & $2 s^{2} 2 p\left({ }^{2} p^{0}\right) 6 p^{3} S$ & $-2.579[-2]$ & $2 s^{2} 2 p\left({ }^{2} P^{0}\right) 8 f^{3} G$ & $-1.563[-2]$ \\
\hline $2 s^{2} 2 p\left({ }^{2} P^{o}\right) 3 p^{1} S$ & $-1.527[-1]$ & $2 s^{2} 2 p\left({ }^{2} P^{0}\right) 6 p^{3} P$ & $-3.529[-2]$ & $2 s^{2} 2 p\left({ }^{2} P^{o}\right) 8 f^{1} G$ & $-1.563[-2]$ \\
\hline $2 s 2 p^{33} p^{0}$ & $-1.522[-1]$ & $2 s^{2} 2 p\left({ }^{2} P^{0}\right) 6 p{ }^{1} D$ & $-3.430[-2]$ & $2 s^{2} 2 p\left({ }^{2} P^{\circ}\right) 8 d^{1} P^{\circ}$ & $-1.563[-2]$ \\
\hline $2 s^{2} 2 p\left({ }^{2} P^{\circ}\right) 3 d^{1} D^{\circ}$ & $-1.221[-1]$ & $2 s^{2} 2 p\left({ }^{2} P^{0}\right) 6 p{ }^{1} S$ & $-3.306[-2]$ & $2 s^{2} 2 p\left({ }^{2} P^{0}\right) 8 f^{3} D$ & $-1.561[-2]$ \\
\hline $2 s^{2} 2 p\left({ }^{2} P^{0}\right) 4 s^{3} P^{0}$ & $-1.191[-1]$ & $2 s^{2} 2 p\left({ }^{2} P^{0}\right) 6 d^{1} D^{\circ}$ & $-2.971[-2]$ & $2 s^{2} 2 p\left({ }^{2} P^{o}\right) 8 f^{1} D$ & $-1.561[-2]$ \\
\hline $2 s^{2} 2 p\left({ }^{2} P^{o}\right) 4 s^{1} P^{o}$ & $-1.173[-1]$ & $2 s^{2} 2 p\left({ }^{2} P^{o}\right) 6 d^{3} F^{o}$ & $-2.871[-2]$ & $2 s^{2} 2 p\left({ }^{2} P^{o}\right) 9 p p^{1} P$ & $-1.473[-2]$ \\
\hline $2 s^{2} 2 p\left({ }^{2} P^{o}\right) 3 d^{3} F^{0}$ & $-1.165[-1]$ & $2 s^{2} 2 p\left({ }^{2} P^{0}\right) 7 s^{3} P^{0}$ & $-2.853[-2]$ & $2 s^{2} 2 p\left({ }^{2} P^{o}\right) 9 p{ }^{3} D$ & $-1.463[-2]$ \\
\hline $2 s^{2} 2 p\left({ }^{2} P^{0}\right) 3 d^{3} D^{\circ}$ & $-1.151[-1]$ & $2 s^{2} 2 p\left({ }^{2} P^{\circ}\right) 6 d^{3} D^{\circ}$ & $-2.838[-2]$ & $2 s^{2} 2 p\left({ }^{2} P^{\circ}\right) 9 p{ }^{3} S$ & $-1.452[-2]$ \\
\hline $2 s^{2} 2 p\left({ }^{2} P^{\circ}\right) 3 d^{1} F^{\circ}$ & $-1.132[-1]$ & $2 s^{2} 2 p\left({ }^{2} P^{o}\right) 7 s^{1} P^{o}$ & $-2.832[-2]$ & $2 s^{2} 2 p\left({ }^{2} P^{0}\right) 9 p{ }^{3} P$ & $-1.440[-2]$ \\
\hline 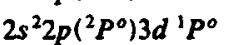 & $-1.110[-1]$ & $2 s^{2} 2 p\left({ }^{2} P^{0}\right) 6 d^{1} F^{\circ}$ & $-2.810[-2]$ & $2 s^{2} 2 p\left({ }^{2} P^{o}\right) 9 p p^{1} D$ & $-1.414[-2]$ \\
\hline $2 s^{2} 2 p\left({ }^{2} P^{0}\right) 3 d^{3} P^{0}$ & $-1.071[-1]$ & $2 s^{2} 2 p\left({ }^{2} P^{0}\right) 6 f^{1} F$ & $-2.790[-2]$ & $2 s^{2} 2 p\left({ }^{2} p^{0}\right) 9 p^{1} s$ & $-1.383[-2]$ \\
\hline $2 s^{2} 2 p\left({ }^{2} P^{0}\right) 4 p^{1} P$ & $-9.875[-2]$ & $2 s^{2} 2 p\left({ }^{2} P^{0}\right) 6 f^{3} F$ & $-2.789[-2]$ & $2 s^{2} 2 p\left({ }^{2} P^{\circ}\right) 9 d^{1} D^{\circ}$ & $-1.296[-2]$ \\
\hline $2 s^{2} 2 p\left({ }^{2} P^{0}\right) 4 p{ }^{3} D$ & $-9.687[-2]$ & $2 s^{2} 2 p\left({ }^{2} P^{0}\right) 6 f^{3} G$ & $-2.779[-2]$ & $2 s^{2} 2 p\left({ }^{2} P^{o}\right) 9 d^{3} F^{o}$ & $-1.263[-2]$ \\
\hline $2 s^{2} 2 p\left({ }^{2} p^{0}\right) 4 p^{3} S$ & $-9.457[-2]$ & $2 s^{2} 2 p\left({ }^{2} P^{0}\right) 6 f^{1} G$ & $-2.779[-2]$ & $2 s^{2} 2 p\left({ }^{2} P^{0}\right) 10 s^{3} P^{0}$ & $-1.256[-2]$ \\
\hline $2 s^{2} 2 p\left({ }^{2} P^{0}\right) 4 p^{3} P$ & $-9.181[-2]$ & $2 s^{2} 2 p\left({ }^{2} P^{o}\right) 6 d^{1} P^{o}$ & $-2.779[-2]$ & $2 s^{2} 2 p\left({ }^{2} P^{\circ}\right) 9 d^{3} D^{\circ}$ & $-1.253[-2]$ \\
\hline $2 s^{2} 2 p\left({ }^{2} P^{0}\right) 4 p^{1} D$ & $-8.731[-2]$ & $2 s^{2} 2 p\left({ }^{2} P^{0}\right) 6 d^{3} P^{0}$ & $-2.778[-2]$ & $2 s^{2} 2 p\left({ }^{2} P^{\circ}\right) 10 s^{3} P^{o}$ & $-1.250[-2]$ \\
\hline $2 s^{2} 2 p\left({ }^{2} P^{0}\right) 4 p{ }^{1} S$ & $-8.161[-2]$ & $2 s^{2} 2 p\left({ }^{2} P^{0}\right) 6 f^{3} D$ & $-2.775[-2]$ & $2 s^{2} 2 p\left({ }^{2} P^{\circ}\right) 9 d^{1} F^{\circ}$ & $-1.244[-2]$ \\
\hline $2 s^{2} 2 p\left({ }^{2} P^{0}\right) 4 d^{1} D^{\circ}$ & $-6.815[-2]$ & $2 s^{2} 2 p\left({ }^{2} P^{0}\right) 6 f^{1} D$ & $-2.775[-2]$ & $2 s^{2} 2 p\left({ }^{2} P^{\circ}\right) 9 f^{1} F$ & $-1.239[-2]$ \\
\hline $2 s^{2} 2 p\left({ }^{2} P^{0}\right) 5 s^{3} P^{\circ}$ & $-6.538[-2]$ & $2 s^{2} 2 p\left({ }^{2} P^{o}\right) 7 p^{1} P$ & $-2.574[-2]$ & $2 s^{2} 2 p\left({ }^{2} P^{\circ}\right) 9 f^{3} F$ & $-1.238[-2]$ \\
\hline $2 s^{2} 2 p\left({ }^{2} P^{0}\right) 4 d^{3} F^{0}$ & $-6.535[-2]$ & $2 s^{2} 2 p\left({ }^{2} P^{o}\right) 7 p^{3} D$ & $-2.552[-2]$ & $2 s^{2} 2 p\left({ }^{2} P^{0}\right) 9 d^{3} P^{o}$ & $-1.236[-2]$ \\
\hline $2 s^{2} 2 p\left({ }^{2} P^{0}\right) 5 s^{1} P^{0}$ & $-6.459[-2]$ & $2 s^{2} 2 p\left({ }^{2} P^{0}\right) 7 p{ }^{3} S$ & $-2.525[-2]$ & $2 s^{2} 2 p\left({ }^{2} P^{0}\right) 9 f^{3} G$ & $-1.235[-2]$ \\
\hline $2 s^{2} 2 p\left({ }^{2} P^{\circ}\right) 4 d^{3} D^{\circ}$ & $-6.441[-2]$ & $2 s^{2} 2 p\left({ }^{2} P^{0}\right) 7 p^{3} P$ & $-2.497[-2]$ & $2 s^{2} 2 p\left({ }^{2} p^{0}\right) 9 f^{1} G$ & $-1.235[-2]$ \\
\hline $2 s^{2} 2 p\left({ }^{2} P^{0}\right) 4 d^{1} F^{0}$ & $-6.351[-2]$ & $2 s^{2} 2 p\left({ }^{2} P^{0}\right) 7 p{ }^{1} D$ & $-2.438[-2]$ & $2 s^{2} 2 p\left({ }^{2} P^{\circ}\right) 9 d^{1} P^{o}$ & $-1.235[-2]$ \\
\hline $2 s^{2} 2 p\left({ }^{2} P^{o}\right) 4 f^{1} F$ & $-6.275[-2]$ & $2 s^{2} 2 p\left({ }^{2} P^{0}\right) 7 p^{1} S$ & $-2.365[-2]$ & $2 s^{2} 2 p\left({ }^{2} P^{0}\right) 9 f^{3} D$ & $-1.234[-2]$ \\
\hline $2 s^{2} 2 p\left({ }^{2} P^{0}\right) 4 f^{3} F$ & $-6.275[-2]$ & $2 s^{2} 2 p\left({ }^{2} P^{0}\right) 7 d^{1} D^{0}$ & $-2.166[-2]$ & $2 s^{2} 2 p\left({ }^{2} P^{0}\right) 9 f^{1} D$ & $-1.234[-2]$ \\
\hline $2 s^{2} 2 p\left({ }^{2} P^{0}\right) 4 d^{1} P^{0}$ & $-6.254[-2]$ & $2 s^{2} 2 p\left({ }^{2} P^{o}\right) 7 d^{3} F^{\circ}$ & $-2.100[-2]$ & $2 s^{2} 2 p\left({ }^{2} P^{0}\right) 10 p^{1} P$ & $-1.171[-2]$ \\
\hline $2 s^{2} 2 p\left({ }^{2} P^{0}\right) 4 f^{3} G$ & $-6.252[-2]$ & $2 s^{2} 2 p\left({ }^{2} P^{o}\right) 8 s^{3} p^{\circ}$ & $-2.087[-2]$ & $2 s^{2} 2 p\left({ }^{2} P^{o}\right) 10 p^{3} D$ & $-1.164[-2]$ \\
\hline $2 s^{2} 2 p\left({ }^{2} P^{0}\right) 4 f^{1} G$ & $-6.252[-2]$ & $2 s^{2} 2 p\left({ }^{2} P^{\circ}\right) 7 d^{3} D^{\circ}$ & $-2.079[-2]$ & $2 s^{2} 2 p\left({ }^{2} P^{\circ}\right) 10 p^{3} S$ & $-1.156[-2]$ \\
\hline $2 s^{2} 2 p\left({ }^{2} P^{o}\right) 4 f^{3} D$ & $-6.244[-2]$ & $2 s^{2} 2 p\left({ }^{2} P^{\circ}\right) 8 s^{1} P^{o}$ & $-2.074[-2]$ & $2 s^{2} 2 p\left({ }^{2} P^{\circ}\right) 10 p{ }^{3} P$ & $-1.148[-2]$ \\
\hline $2 s^{2} 2 p\left({ }^{2} P^{o}\right) 4 f^{1} D$ & $-6.244[-2]$ & $2 s^{2} 2 p\left({ }^{2} P^{\circ}\right) 7 d^{1} F^{\circ}$ & $-2.061[-2]$ & $2 s^{2} 2 p\left({ }^{2} P^{\circ}\right) 10 p p^{1} D$ & $-1.129[-2]$ \\
\hline $2 s^{2} 2 p\left({ }^{2} P^{0}\right) 4 d^{3} P^{0}$ & $-6.206[-2]$ & $2 s^{2} 2 p\left({ }^{2} P^{0}\right) 7 f^{1} F$ & $-2.049[-2]$ & $2 s^{2} 2 p\left({ }^{2} P^{o}\right) 10 p p^{1} S$ & $-1.107[-2]$ \\
\hline $2 s^{2} 2 p\left({ }^{2} P^{o}\right) 5 p p^{1} P$ & $-5.638[-2]$ & $2 s^{2} 2 p\left({ }^{2} P^{0}\right) 7 f^{3} F$ & $-2.049[-2]$ & $2 s^{2} 2 p\left({ }^{2} P^{\circ}\right) 10 d^{1} D^{\circ}$ & $-1.045[-2]$ \\
\hline $2 s^{2} 2 p\left({ }^{2} P^{0}\right) 5 p^{3} D$ & $-5.563[-2]$ & $2 s^{2} 2 p\left({ }^{2} P^{0}\right) 7 d^{3} P^{o}$ & $-2.042[-2]$ & $2 s^{2} 2 p\left({ }^{2} P^{0}\right) 10 d^{3} F^{\circ}$ & $-1.021[-2]$ \\
\hline $2 s^{2} 2 p\left({ }^{2} P^{0}\right) 5 p^{3} S$ & $-5.474[-2]$ & $2 s^{2} 2 p\left({ }^{2} P^{\circ}\right) 7 f^{3} G$ & $-2.042[-2]$ & $2 s^{2} 2 p\left({ }^{2} P^{o}\right) 11 s^{3} P^{a}$ & $-1.015[-2]$ \\
\hline $2 s^{2} 2 p\left({ }^{2} P^{0}\right) 5 p{ }^{3} P$ & $-5.372[-2]$ & $2 s^{2} 2 p\left({ }^{2} P^{0}\right) 7 f^{1} G$ & $-2.042[-2]$ & $2 s^{2} 2 p\left({ }^{2} P^{o}\right) 10 d^{3} D^{o}$ & $-1.013[-2]$ \\
\hline $2 s^{2} 2 p\left({ }^{2} P^{0}\right) 5 p^{\prime} D$ & $-5.183[-2]$ & $2 s^{2} 2 p\left({ }^{2} P^{\circ}\right) 7 d^{1} P^{\circ}$ & $-2.041[-2]$ & $2 s^{2} 2 p\left({ }^{2} P^{o}\right) 11 s^{1} P^{o}$ & $-1.011[-2]$ \\
\hline $2 s^{2} 2 p\left({ }^{2} P^{0}\right) 5 p p^{1} S$ & $-4.943[-2]$ & $2 s^{2} 2 p\left({ }^{2} P^{0}\right) 7 f^{3} D$ & $-2.039[-2]$ & $2 s^{2} 2 p\left({ }^{2} P^{\circ}\right) 10 d^{1} F^{\circ}$ & $-1.007[-2]$ \\
\hline $2 s^{2} 2 p\left({ }^{2} P^{0}\right) S d^{1} D^{0}$ & $-4.317[-2]$ & $2 s^{2} 2 p\left({ }^{2} P^{0}\right) 7 f^{1} D$ & $-2.039[-2]$ & $2 s^{2} 2 p\left({ }^{2} P^{\circ}\right) 10 f^{1} F$ & $-1.003[-2]$ \\
\hline
\end{tabular}


TABLE II. (Continued).

\begin{tabular}{cccccc}
\hline \hline State & $E$ & State & $E$ & State \\
\hline & & & & & \\
& & & & & \\
$2 s^{2} 2 p\left({ }^{2} P^{o}\right) 5 d^{3} F^{o}$ & $-4.156[-2]$ & $2 s^{2} 2 p\left({ }^{2} P^{o}\right) 8 p^{1} P$ & $-1.909[-2]$ & $2 s^{2} 2 p\left({ }^{2} P^{\circ}\right) 10 f^{3} F$ & $-1.003[-2]$ \\
$2 s^{2} 2 p\left({ }^{2} P^{o}\right) 6 s^{3} P^{o}$ & $-4.136[-2]$ & $2 s^{2} 2 p\left({ }^{2} P^{o}\right) 8 p^{3} D$ & $-1.895[-2]$ & $2 s^{2} 2 p\left({ }^{2} P^{0}\right) 10 d^{3} P^{\circ}$ & $-1.001[-2]$ \\
$2 s^{2} 2 p\left({ }^{2} P^{o}\right) 5 d^{3} D^{o}$ & $-4.102[-2]$ & $2 s^{2} 2 p\left({ }^{2} P^{o}\right) 8 p^{3} S$ & $-1.878[-2]$ & $2 s^{2} 2 p\left({ }^{2} P^{o}\right) 10 f^{3} G$ & $-1.000[-2]$ \\
$2 s^{2} 2 p\left({ }^{2} P^{0}\right) 6 s^{1} P^{o}$ & $-4.097[-2]$ & $2 s^{2} 2 p\left({ }^{2} P^{o}\right) 8 p^{3} P$ & $-1.861[-2]$ & $2 s^{2} 2 p\left({ }^{2} P^{o}\right) 10 f^{1} G$ & $-1.000[-2]$
\end{tabular}

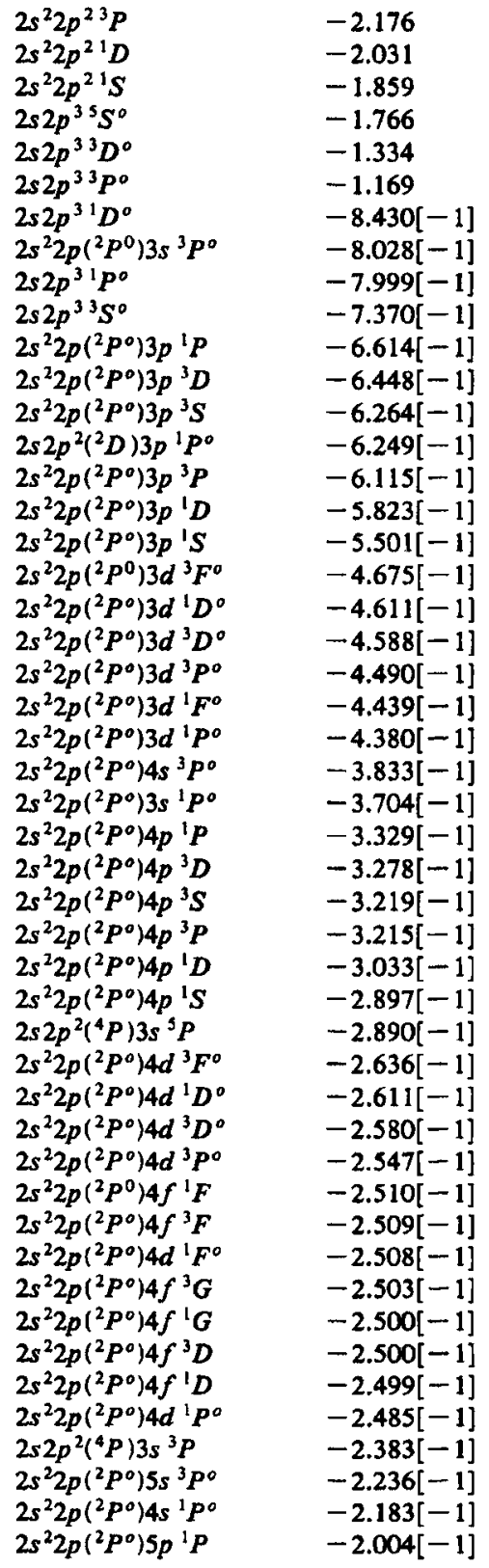

\begin{tabular}{|c|c|}
\hline \multicolumn{2}{|c|}{$\mathbf{N}^{+}$} \\
\hline$s^{2} 2 p\left({ }^{2} P^{o}\right) 5 f^{1} F$ & $-1.608[-1]$ \\
\hline $2 s^{2} 2 p\left(^{2} p^{0}\right) 5 f^{3} F$ & $-1.607[-1]$ \\
\hline $2 s^{2} 2 p\left({ }^{2} P^{0}\right) 5 f^{3} G$ & $-1.602[-1]$ \\
\hline $2 s^{2} 2 p\left({ }^{2} P^{0}\right) 5 f^{1} G$ & $-1.600[-1]$ \\
\hline $2 s^{2} 2 p\left({ }^{2} P^{o}\right) 5 f^{3} D$ & $-1.600[-1]$ \\
\hline $2 s^{2} 2 p\left({ }^{2} P^{\circ}\right) 5 f^{1} D$ & $-1.599[-1]$ \\
\hline $2 s^{2} 2 p\left({ }^{2} P^{\circ}\right) 5 d^{1} P^{\circ}$ & $-1.596[-1]$ \\
\hline $2 s 2 p^{2}\left({ }^{4} P\right) 3 d^{3} P$ & $-1.594[-1]$ \\
\hline $2 s^{2} 2 p\left({ }^{2} P^{0}\right) 6 s^{3} P^{0}$ & $-1.464[-1]$ \\
\hline $2 s^{2} 2 p\left(^{2} P^{o}\right) 5 s^{1} P^{\circ}$ & $-1.435[-1]$ \\
\hline $2 s^{2} 2 p\left(^{2} P^{0}\right) 6 p^{1} P$ & $-1.337[-1]$ \\
\hline $2 s^{2} 2 p\left({ }^{2} p^{0}\right) 6 p^{3} D$ & $-1.326[-1]$ \\
\hline $2 s^{2} 2 p\left({ }^{2} P^{o}\right) 6 p{ }^{3} S$ & $-1.311[-1]$ \\
\hline $2 s 2 p^{2}\left({ }^{4} P\right) 3 p^{3} S^{0}$ & $-1.302[-1]$ \\
\hline $2 s^{2} 2 p\left({ }^{2} P^{0}\right) 6 p{ }^{3} P$ & $-1.262[-1]$ \\
\hline $2 s^{2} 2 p\left({ }^{2} p^{0}\right) 6 p{ }^{1} D$ & $-1.261[-1]$ \\
\hline $2 s 2 p^{2}\left({ }^{4} P\right) 3 p^{5} D^{0}$ & $-1.258[-1]$ \\
\hline $2 s^{2} 2 p\left({ }^{2} P^{0}\right) 6 p{ }^{1} S$ & $-1.222[-1]$ \\
\hline $2 s^{2} 2 p\left({ }^{2} P^{o}\right) 6 d^{3} F^{0}$ & $-1,158[-1]$ \\
\hline $2 s^{2} 2 p\left({ }^{2} P^{\circ}\right) 6 d^{1} D^{\circ}$ & $-1.151[-1]$ \\
\hline $2 s^{2} 2 p\left({ }^{2} P^{\circ}\right) 6 d^{3} D^{\circ}$ & $-1.143[-1]$ \\
\hline $2 s^{2} 2 p\left({ }^{2} P^{o}\right) 6 d^{3} P^{o}$ & $-1.133[-1]$ \\
\hline $2 s^{2} 2 p\left({ }^{2} P^{o}\right) 6 d^{1} F^{o}$ & $-1.117[-1]$ \\
\hline $2 s^{2} 2 p\left(^{2} P^{o}\right) 6 f^{1} F$ & $-1.116[-1]$ \\
\hline $2 s^{2} 2 p\left({ }^{2} P^{\circ}\right) 6 f^{3} F$ & $-1.116[-1]$ \\
\hline $2 s^{2} 2 p\left({ }^{2} P^{0}\right) 6 f^{3} G$ & $-1.113[-1]$ \\
\hline $2 s^{2} 2 p\left({ }^{2} P^{0}\right) 6 f^{1} G$ & $-1.111[-1]$ \\
\hline $2 s^{2} 2 p\left({ }^{2} P^{0}\right) 6 f^{3} D$ & $-1.111[-1]$ \\
\hline $2 s^{2} 2 p\left({ }^{2} P^{0}\right) 6 f^{1} D$ & $-1.111[-1]$ \\
\hline $2 s^{2} 2 p\left({ }^{2} p^{0}\right) 6 d^{1} p^{\circ}$ & $-1.110[-1]$ \\
\hline $2 s 2 p^{2}\left({ }^{4} P\right) 3 p^{5} P^{0}$ & $-1.098[-1[$ \\
\hline $2 s^{2} 2 p\left({ }^{2} P^{0}\right) 7 s^{3} P^{0}$ & $-1.032[-1]$ \\
\hline $2 s^{2} 2 p\left({ }^{2} P^{o}\right) 6 s^{1} P^{o}$ & $-1.015[-1]$ \\
\hline $2 s^{2} 2 p\left({ }^{2} P^{0}\right) 7 p^{1} P$ & $-9.557[-2]$ \\
\hline $2 s^{2} 2 p\left({ }^{2} P^{0}\right) 7 p^{3} D$ & $-9.492[-2]$ \\
\hline $2 s^{2} 2 p\left({ }^{2} P^{o}\right) 7 p^{3} S$ & $-9.401[-2]$ \\
\hline $2 s^{2} 2 p\left({ }^{2} P^{0}\right) 7 p p^{3} P$ & $-9.158[-2]$ \\
\hline $2 s^{2} 2 p\left({ }^{2} P^{o}\right) 7 p{ }^{1} D$ & $-9.100[-2]$ \\
\hline $2 s^{2} 2 p\left({ }^{2} P^{0}\right) 7 p{ }^{1} S$ & $-8.852[-2]$ \\
\hline $2 s^{2} 2 p\left({ }^{2} P^{0}\right) 7 d^{3} F^{\circ}$ & $-8.467[-2]$ \\
\hline $2 s^{2} 2 p\left({ }^{2} P^{0}\right) 7 d^{3} D^{0}$ & $-8.455[-2]$ \\
\hline $2 s^{2} 2 p\left({ }^{2} P^{0}\right) 7 d^{1} D^{\circ}$ & $-8.425[-2]$ \\
\hline $2 s^{2} 2 p\left({ }^{2} P^{\circ}\right) 7 d^{3} P^{o}$ & $-8.324[-2]$ \\
\hline $2 s^{2} 2 p\left({ }^{2} P^{0}\right) 7 d^{1} F^{o}$ & $-8.205[-2]$ \\
\hline $2 s^{2} 2 p\left({ }^{2} P^{0}\right) 7 f^{1} F$ & $-8.199[-2]$ \\
\hline${ }^{2} 2 p\left({ }^{2} P^{0}\right) 7 f^{3} F$ & $-8.197[-2]$ \\
\hline$p\left({ }^{2} P^{0}\right) 7 f^{3} G$ & $-8.175[-2]$ \\
\hline${ }^{2} 2 p\left({ }^{2} P^{o}\right) 7 f^{1} G$ & $-8.166[-2]$ \\
\hline
\end{tabular}

$2 s^{2} 2 p\left({ }^{2} P^{\circ}\right) 8 p{ }^{3} S$ $2 s^{2} 2 p\left({ }^{2} P^{\circ}\right) 8 p{ }^{3} P$ $2 s^{2} 2 p\left({ }^{2} P^{\circ}\right) 8 p^{1} D$ $2 s^{2} 2 p\left({ }^{2} P^{0}\right) 8 p{ }^{1} S$ $2 s^{2} 2 p\left({ }^{2} P^{0}\right) 8 d^{3} F^{\circ}$ $2 s^{2} 2 p\left({ }^{2} P^{0}\right) 8 d^{1} D^{\circ}$ $2 s^{2} 2 p\left({ }^{2} P^{\circ}\right) 8 d^{3} P^{o}$ $2 s^{2} 2 p\left({ }^{2} P^{\circ}\right) 8 d^{3} D^{\circ}$ $2 s^{2} 2 p\left({ }^{2} P^{0}\right) 8 d^{1} F^{\circ}$ $2 s^{2} 2 p\left({ }^{2} P^{o}\right) 8 f^{1} F$ $2 s^{2} 2 p\left({ }^{2} P^{o}\right) 8 f^{3} F$ $2 s^{2} 2 p\left({ }^{2} P^{\circ}\right) 8 f^{3} G$ $2 s^{2} 2 p\left({ }^{2} P^{0}\right) 8 f^{1} G$ $2 s^{2} 2 p\left({ }^{2} P^{\circ}\right) 8 d^{1} P^{0}$ $2 s^{2} 2 p\left({ }^{2} P^{\circ}\right) 8 f^{3} D$ $\left.2 s^{2} 2 p^{2}{ }^{2} P^{\circ}\right) 8 f^{1} D$ $2 s^{2} 2 p\left({ }^{2} P^{0}\right) 9 s^{3} P^{0}$ $2 s^{2} 2 p\left({ }^{2} P^{0}\right) 8 s^{1} P^{\circ}$ $2 s^{2} 2 p\left({ }^{2} P^{\circ}\right) 9 p^{1} P$ $2 s^{2} 2 p\left({ }^{2} P^{\circ}\right) 9 p{ }^{3} D$ $2 s^{2} 2 p\left({ }^{2} P^{0}\right) 9 p{ }^{3} S$ $2 s^{2} 2 p\left(^{2} P^{0}\right) 9 p{ }^{3} P$ $\left.2 s^{2} 2 p^{2} P^{\circ}\right) 9 p^{1} D$ $2 s 2 p^{2}\left({ }^{4} P\right) 3 p^{3} p^{\circ}$ $2 s^{2} 2 p\left({ }^{2} P^{0}\right) 9 p{ }^{1} S$ $2 s^{2} 2 p\left({ }^{2} P^{0}\right) 9 d^{3} F^{\circ}$ $2 s^{2} 2 p\left({ }^{2} P^{o}\right) 9 d^{1} D^{\circ}$ $2 s^{2} 2 p\left({ }^{2} p^{\circ}\right) 9 d^{3} D^{\circ}$ $2 s^{2} 2 p\left({ }^{2} P^{o}\right) 9 d^{1} F^{o}$ $2 s^{2} 2 p\left({ }^{2} P^{0}\right) 9 f^{1} F$ $2 s^{2} 2 p\left({ }^{2} P^{o}\right) 9 f^{3} F$ $2 s^{2} 2 p\left({ }^{2} P^{\circ}\right) 9 f^{3} G$ $2 s^{2} 2 p\left({ }^{2} P^{\circ}\right) 9 d^{1} P^{\circ}$ $2 s^{2} 2 p\left({ }^{2} P^{\circ}\right) 9 f^{\prime} G$ $2 s^{2} 2 p\left({ }^{2} P^{o}\right) 9 f^{3} D$ $2 s^{2} 2 p\left({ }^{2} P^{\circ}\right) 9 f^{1} D$ $2 s^{2} 2 p\left({ }^{2} P^{o}\right) 9 d^{3} p^{\circ}$ $2 s^{2} 2 p\left({ }^{2} P^{o}\right) 9 s^{1} P^{\circ}$ $2 s^{2} 2 p\left({ }^{2} P^{o}\right) 10 s^{3} P^{o}$ $2 s^{2} 2 p\left({ }^{2} P^{0}\right) 10 p{ }^{1} P$ $2 s^{2} 2 p\left({ }^{2} P^{\circ}\right) 10 p{ }^{3} D$ $2 s^{2} 2 p\left({ }^{2} P^{0}\right) 10 p^{3} S$ $2 s^{2} 2 p\left({ }^{2} P^{o}\right) 10 p^{3} P$ $2 s^{2} 2 p\left({ }^{2} P^{o}\right) 10 p^{1} D$ $2 s^{2} 2 p\left({ }^{2} p^{0}\right) 10 p{ }^{1} S$ $2 s^{2} 2 p\left({ }^{2} P^{\circ}\right) 10 d^{3} F^{\circ}$ $2 s^{2} 2 p\left({ }^{2} P^{\circ}\right) 10 d^{1} D^{\circ}$ $2 s^{2} 2 p\left({ }^{2} P^{o}\right) 10 d^{3} D^{\circ}$

$$
\begin{aligned}
& -7.068[-2] \\
& -6.922[-2] \\
& -6.867[-2] \\
& -6.707[-2] \\
& -6.456[-2] \\
& -6.429[-2] \\
& -6.394[-2] \\
& -6.301[-2] \\
& -6.280[-2] \\
& -6.275[-2] \\
& -6.274[-2] \\
& -6.259[-2] \\
& -6.252[-2] \\
& -6.252[-2] \\
& -6.252[-2] \\
& -6.247[-2] \\
& -5.952[-2] \\
& -5.837[-2] \\
& -5.575[-2] \\
& -5.549[-2] \\
& -5.507[-2] \\
& -5.411[-2] \\
& -5.370[-3] \\
& -5.261[-2] \\
& -5.257[-2] \\
& -5.085[-2] \\
& -5.065[-2] \\
& -5.000[-2] \\
& -4.960[-2] \\
& -4.957[-2] \\
& -4.955[-2] \\
& -4.945[-2] \\
& -4.941[-2] \\
& -4.940[-2] \\
& -4.940[-2] \\
& -4.937[-2] \\
& -4.888[-2] \\
& -4.647[-2] \\
& -4.622[-2] \\
& -4.460[-2] \\
& -4.441[-2] \\
& -4.411[-2] \\
& -4.344[-2] \\
& -4.313[-2] \\
& -4.231[-2] \\
& -4.107[-2] \\
& -4.093[-2] \\
& -4.050[-2]
\end{aligned}
$$


TABLE II. (Continued).

\begin{tabular}{|c|c|c|c|c|c|}
\hline State & $E$ & State & $E$ & State & $E$ \\
\hline $\begin{array}{l}2 s^{2} 2 p\left({ }^{2} P^{o}\right) 5 p{ }^{3} D \\
2 s^{2} 2 p\left({ }^{2} P^{o}\right) 5 p{ }^{3} S \\
2 s^{2} 2 p\left({ }^{2} P^{o}\right) 5 p{ }^{3} P \\
2 s^{2} 2 p\left({ }^{2} P^{\circ}\right) 5 p{ }^{1} D \\
2 s^{2} 2 p\left({ }^{2} P^{o}\right) 5 p \\
2 s^{2} 2 p\left({ }^{2} P^{o}\right) 5 d^{3} F^{o} \\
2 s^{2} 2 p\left({ }^{2} P^{o}\right) 5 d^{1} D^{\circ} \\
2 s^{2} 2 p\left({ }^{2} P^{o}\right) 5 d^{3} D^{o} \\
2 s^{2} 2 p\left({ }^{2} P^{\circ}\right) 5 d^{3} P^{o} \\
2 s^{2} 2 p\left({ }^{2} P^{o}\right) 5 d^{1} F^{o}\end{array}$ & $\begin{array}{l}-1.982[-1] \\
-1.955[-1] \\
-1.871[-1] \\
-1.865[-1] \\
-1.796[-1] \\
-1.678[-1] \\
-1.666[-1] \\
-1.648[-1] \\
-1.632[-1] \\
-1.608[-1]\end{array}$ & $\begin{array}{l}2 s^{2} 2 p\left({ }^{2} P^{o}\right) 7 f^{3} D \\
2 s^{2} 2 p\left({ }^{2} P^{\circ}\right) 7 d^{1} P^{\circ} \\
2 s^{2} 2 p\left({ }^{2} P^{o}\right) 7 f^{1} D \\
2 s^{2} 2 p\left({ }^{2} P^{\circ}\right) 8 s^{3} P^{\circ} \\
2 s 2 p^{2}\left({ }^{2} D\right) 3 s^{1} D \\
2 s^{2} 2 p\left({ }^{2} P^{\circ}\right) 7 s^{1} P^{\circ} \\
2 s 2 p^{2}\left({ }^{4} P\right) 3 p{ }^{5} S^{o} \\
2 s^{2} p^{2}\left({ }^{4} P\right) 3 p^{3} D^{\circ} \\
2 s^{2} 2 p\left({ }^{2} P^{\circ}\right) 8 p^{1} P\end{array}$ & $\begin{array}{l}-8.165[-2] \\
-8.163[-2] \\
-8.163[-2] \\
-7.676[-2] \\
-7.664[-2] \\
-7.551[-2] \\
-7.495[-2] \\
-7.417[-2] \\
-7.168[-2]\end{array}$ & $\begin{array}{l}2 s^{2} 2 p\left({ }^{2} P^{o}\right) 10 d^{3} P^{o} \\
2 s^{2} 2 p\left({ }^{2} P^{o}\right) 10 d^{1} F^{o} \\
2 s^{2} 2 p\left({ }^{2} P^{o}\right) 10 f^{1} F \\
2 s^{2} 2 p\left({ }^{2} P^{o}\right) 10 f^{3} F \\
2 s^{2} 2 p\left({ }^{2} P^{o}\right) 10 f^{3} G \\
2 s^{2} 2 p\left({ }^{2} P^{o}\right) 10 d^{1} P^{\circ} \\
2 s^{2} 2 p\left({ }^{2} P^{o}\right) 10 f^{3} D \\
2 s^{2} 2 p\left({ }^{2} P^{o}\right) 10 f^{1} G\end{array}$ & $\begin{array}{l}-4.020[-2] \\
-4.017[-2] \\
-4.014[-2] \\
-4.013[-2] \\
-4.005[-2] \\
-4.002[-2] \\
-4.001[-2] \\
-4.000[-2]\end{array}$ \\
\hline
\end{tabular}

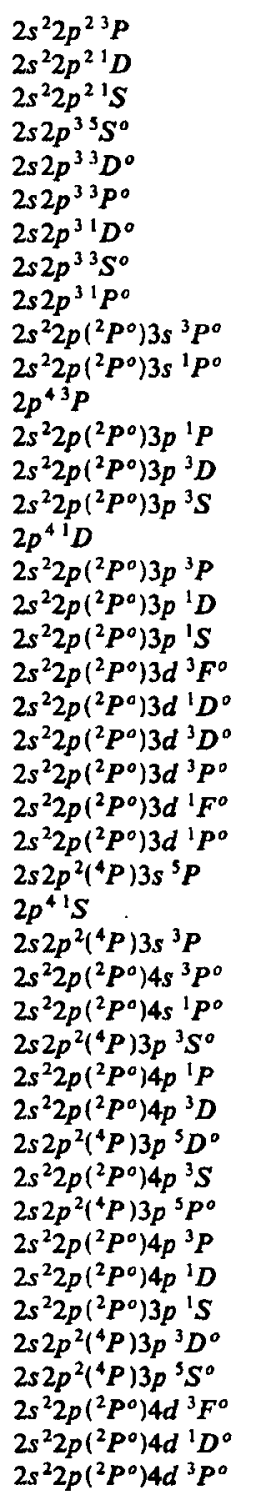

$-4.036$

$-3.847$

$-3.625$

$-3.507$

$-2.944$

$-2.729$

$-2.311$

$-2.214$

$-2.085$

$-1.581$

$-1.529$

$-1.442$

$-1.369$

$-1.342$

$-1.311$

$-1.306$

$-1.288$

$-1.234$

$-1.171$

$-1.069$

$-1.068$

$-1.044$

$-1.025$

$-1.004$

$-9.935[-1]$

$-9.369[-1]$

$-8.747[-1]$

$-8.363[-1]$

$-7.884[-1]$

$-7.707[-1]$

$-7.146[-1]$

$-7.066[-1]$

$-6.995[-1]$

$-6.934[-1]$

$-6.891[-1]$

$-6.676[-1]$

$-6.604[-1]$

$-6.571[-1]$

$-6.299[-1]$

$-6.149[-1]$

$-6.047[-1]$

$-5.983[-1]$

$-5.962[-1]$

$-5.873[-1]$
$\mathrm{O}^{2+}$

\begin{tabular}{|c|c|}
\hline & \\
\hline $2 s^{2} 2 p\left(^{2} P^{o}\right) 4 p^{1} S$ & $-3.938[-1]$ \\
\hline $2 s 2 p^{2}\left({ }^{4} P\right) 3 d^{3} P$ & $-3.806[-1]$ \\
\hline $2 s^{2} 2 p\left({ }^{2} P^{\circ}\right) 5 d^{3} F^{o}$ & $-3.793[-1]$ \\
\hline $2 s^{2} 2 p\left({ }^{2} P^{o}\right) 5 d^{1} D^{o}$ & $-3.784[-1]$ \\
\hline $2 s 2 p^{2}\left({ }^{4} P\right) 3 d{ }^{3} F$ & $-3.712[-1]$ \\
\hline $2 s^{2} 2 p\left({ }^{2} P^{o}\right) 5 d^{3} D^{\circ}$ & $-3.712[-1]$ \\
\hline $2 s^{2} 2 p\left({ }^{2} P^{0}\right) 5 d^{3} P^{0}$ & $-3.700[-1]$ \\
\hline $2 s^{2} 2 p\left(^{2} P^{o}\right) 5 d^{1} F^{o}$ & $-3.635[-1]$ \\
\hline $2 s^{2} 2 p\left({ }^{2} P^{\circ}\right) 5 f^{1} F$ & $-3.631[-1]$ \\
\hline $2 s^{2} 2 p\left({ }^{2} P^{\circ}\right) 4 f^{3} F$ & $-3.625[-1]$ \\
\hline $2 s^{2} 2 p\left({ }^{2} P^{\circ}\right) 5 d^{1} P^{o}$ & $-3.616[-1]$ \\
\hline $2 s^{2} 2 p\left({ }^{2} P^{\circ}\right) 5 f^{3} G$ & $-3.613[-1]$ \\
\hline $2 s^{2} 2 p\left({ }^{2} P^{\circ}\right) S f^{3} D$ & $-3.605[-1]$ \\
\hline $2 s^{2} 2 p\left({ }^{2} P^{\circ}\right) 4 f^{1} G$ & $-3.604[-1]$ \\
\hline $2 s^{2} 2 p\left({ }^{2} P^{0}\right) 5 f^{1} D$ & $-3.600[-1]$ \\
\hline $2 s 2 p^{2}\left({ }^{2} D\right) 3 s^{1} D$ & $-3.336[-1]$ \\
\hline $2 s 2 p^{2}\left({ }^{4} P\right) 3 d^{3} D$ & $-3.314[-1]$ \\
\hline $2 s^{2} 2 p\left({ }^{2} P^{0}\right) 6 s^{3} P^{0}$ & $-3.116[-1]$ \\
\hline $2 s^{2} 2 p\left({ }^{2} P^{0}\right) 6 s^{1} P^{\circ}$ & $-3.071[-1]$ \\
\hline $2 s^{2} 2 p\left({ }^{2} P^{0}\right) 6 p p^{1} P$ & $-2.904[-1]$ \\
\hline $2 s^{2} 2 p\left({ }^{2} p^{0}\right) 6 p{ }^{3} s$ & $-2.863[-1]$ \\
\hline $2 s^{2} 2 p\left({ }^{2} P^{0}\right) 6 p{ }^{3} D$ & $-2.851[-1]$ \\
\hline $2 s^{2} 2 p\left({ }^{2} P^{0}\right) 6 p{ }^{3} P$ & $-2.814[-1]$ \\
\hline $2 s^{2} 2 p\left({ }^{2} P^{o}\right) 6 p{ }^{1} D$ & $-2.735[-1]$ \\
\hline $2 s^{2} 2 p\left({ }^{2} p^{\circ}\right) s p p^{1} S$ & $-2.694[-1]$ \\
\hline $2 s^{2} 2 p\left({ }^{2} p^{o}\right) 6 d^{3} F^{o}$ & $-2.614[-1]$ \\
\hline $2 s^{2} 2 p\left({ }^{2} P^{\circ}\right) 6 d^{1} D^{\circ}$ & $-2.612[-1]$ \\
\hline $2 s^{2} 2 p\left({ }^{2} P^{o}\right) 6 d^{3} D^{0}$ & $-2.567[-1]$ \\
\hline $2 s^{2} 2 p\left({ }^{2} P^{0}\right) 6 d^{3} P^{\circ}$ & $-2.562[-1]$ \\
\hline $2 s^{2} 2 p\left({ }^{2} P^{\circ}\right) 6 d^{1} F^{\circ}$ & $-2.526[-1]$ \\
\hline $2 s^{2} 2 p\left({ }^{2} P^{o}\right) 6 f^{1} F$ & $-2.521[-1]$ \\
\hline $2 s^{2} 2 p\left({ }^{2} P^{o}\right) 5 f^{3} F$ & $-2.519[-1]$ \\
\hline $2 s^{2} 2 p\left({ }^{2} P^{o}\right) 6 d{ }^{1} P^{o}$ & $-2.512[-1]$ \\
\hline $2 s^{2} 2 p\left({ }^{2} P^{o}\right) 6 f^{3} G$ & $-2.510[-1]$ \\
\hline $2 s^{2} 2 p\left(^{2} P^{0}\right) 6 f^{3} D$ & $-2.504[-1]$ \\
\hline $2 s^{2} 2 p\left({ }^{2} p^{o}\right) 5 f^{1} G$ & $-2.503[-1]$ \\
\hline $2 s^{2} 2 p\left({ }^{2} P^{o}\right) 6 f^{1} D$ & $-2.501[-1]$ \\
\hline $2 s^{2} 2 p\left({ }^{2} P^{\circ}\right) 7 s^{3} P^{o}$ & $-2.220[-1]$ \\
\hline $2 s^{2} 2 p\left({ }^{2} P^{o}\right) 7 s^{1} P^{\circ}$ & $-2.189[-1]$ \\
\hline $2 s^{2} 2 p\left({ }^{2} P^{o}\right) 7 p^{1} P$ & $-2.088[-1]$ \\
\hline $2 s^{2} 2 p\left({ }^{2} P^{0}\right) 7 p{ }^{3} s$ & $-2.065[-1]$ \\
\hline $2 s^{2} 2 p\left({ }^{2} P^{\circ}\right) 7 p{ }^{3} D$ & $-2.059[-1]$ \\
\hline $2 s^{2} 2 p\left({ }^{2} P^{o}\right) 7 p{ }^{3} P$ & $-2.039[-1]$ \\
\hline $2 s^{2} 2 p\left({ }^{2} P^{\circ}\right) 7 p{ }^{1} D$ & $-1.993[-1]$ \\
\hline
\end{tabular}

$2 s 2 p^{2}\left({ }^{2} D\right) 3 p^{3} D^{\circ}$ $2 s^{2} 2 p\left({ }^{2} P^{\circ}\right) 8 d^{1} D^{\circ}$ $2 s^{2} 2 p\left({ }^{2} P^{\circ}\right) 7 p$ ' $S$ $2 s^{2} 2 p\left({ }^{2} P^{\circ}\right) 8 d^{3} F^{\circ}$ $2 s^{2} 2 p\left({ }^{2} P^{o}\right) 8 d^{1} F^{o}$ $2 s^{2} 2 p\left({ }^{2} P^{\circ}\right) 8 d^{3} D^{\circ}$ $2 s^{2} 2 p\left({ }^{2} P^{\circ}\right) 8 d^{3} P^{\circ}$ $2 s^{2} 2 p\left({ }^{2} P^{0}\right) 8 f^{1} F$ $2 s^{2} 2 p\left({ }^{2} P^{\circ}\right) 7 f^{3} F$ $2 s^{2} 2 p\left({ }^{2} P^{\circ}\right) 8 d^{1} P^{\circ}$ $2 s^{2} 2 p\left({ }^{2} P^{\circ}\right) 8 f^{3} G$ $2 s^{2} 2 p\left({ }^{2} P^{0}\right) 8 f^{3} D$ $\left.2 s^{2} 2 p^{(2} P^{\circ}\right) 7 f^{1} G$ $2 s^{2} 2 p\left({ }^{2} P^{\circ}\right) 8 f^{1} D$ $2 s 2 p^{2}\left({ }^{2} D\right) 3 p^{1} D^{\circ}$ $2 s 2 p^{2}\left({ }^{4} P\right) 4 s^{5} P$ $2 s 2 p^{2}\left({ }^{2} D\right) 3 p^{1} F^{\circ}$ $2 s^{2} 2 p\left({ }^{2} P^{o}\right) 9 s^{3} P^{o}$ $2 s^{2} 2 p\left({ }^{2} p^{\circ}\right) 9 s^{1} p^{\circ}$ $2 s^{2} 2 p\left({ }^{2} P^{o}\right) 9 p^{1} P$ $2 s^{2} 2 p\left({ }^{2} P^{\circ}\right) 9 p^{3} P$ $2 s^{2} 2 p\left({ }^{2} p^{0}\right) 9 p{ }^{3} S$ $2 s^{2} 2 p\left({ }^{2} p^{\circ}\right) 9 p p^{3} D$ $2 s^{2} 2 p\left({ }^{2} P^{\circ}\right) 9 p p^{1} D$ $2 s^{2} 2 p\left({ }^{2} p^{\circ}\right) 8 p p^{1} S$ $2 s^{2} 2 p\left({ }^{2} P^{\circ}\right) 9 d^{3} F^{\circ}$ $2 s^{2} 2 p\left({ }^{2} P^{\circ}\right) 9 d^{3} P^{\circ}$ $2 s^{2} 2 p\left({ }^{2} P^{\circ}\right) 9 d^{1} D^{\circ}$ $2 s^{2} 2 p\left({ }^{2} P^{\circ}\right) 9 d^{3} D^{\circ}$ $\left.2 s^{2} 2 p^{(2} P^{0}\right) 9 f^{1} F$ $2 s 2 p^{2}\left({ }^{2} D\right) 3 p^{3} P^{o}$ $2 s^{2} 2 p\left({ }^{2} p^{\circ}\right) 9 d^{1} p^{\circ}$ $2 s^{2} 2 p\left({ }^{2} P^{\circ}\right) 8 f^{3} F$ $2 s^{2} 2 p\left({ }^{2} p^{0}\right) 9 f^{3} G$ $2 s^{2} 2 p\left({ }^{2} P^{\circ}\right) 9 f^{3} D$ $2 s^{2} 2 p\left({ }^{2} P^{o}\right) 8 f^{1} G$ $2 s^{2} 2 p\left({ }^{2} P^{\circ}\right) 9 f^{1} D$ $2 s^{2} 2 p\left({ }^{2} P^{\circ}\right) 9 d^{1} F^{\circ}$ $2 s 2 p^{2}\left({ }^{4} P\right) 4 s^{3} P$ $2 s^{2} 2 p\left({ }^{2} P^{\circ}\right) 10 s^{1} P^{\circ}$ $2 s^{2} 2 p\left({ }^{2} P^{\circ}\right) 10 s^{3} P^{\circ}$ $2 s 2 p^{2}\left({ }^{2} D\right) 3 p^{1} P^{\circ}$ $2 s^{2} 2 p\left({ }^{2} P^{0}\right) 10 p^{1} P$ $2 s^{2} 2 p\left({ }^{2} P^{o}\right) 10 p{ }^{3} S$

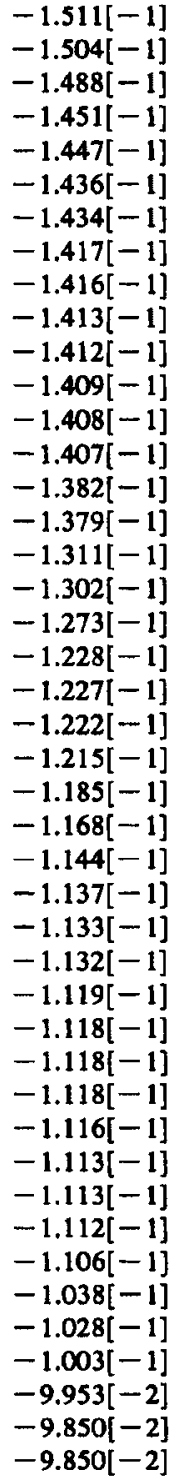


TABLE II. (Continued).

\begin{tabular}{|c|c|c|c|c|c|}
\hline State & $E$ & State & $E$ & State & $E$ \\
\hline $2 s^{2} 2 p\left(^{2} P^{0}\right) 4 d^{3} D^{0}$ & $-5.794[-1]$ & $2 s^{2} 2 p\left({ }^{2} P^{o}\right) 6 p{ }^{1} S$ & $-1.958[-1]$ & $2 s^{2} 2 p\left({ }^{2} P^{o}\right) 10 p^{3} D$ & $-9.752[-2]$ \\
\hline $2 s^{2} 2 p\left({ }^{2} P^{o}\right) 4 d^{1} F^{o}$ & $-5.673[-1]$ & $2 s^{2} 2 p\left({ }^{2} P^{o}\right) 7 d^{1} D^{\circ}$ & $-1.915[-1]$ & $2 s^{2} 2 p\left({ }^{2} P^{o}\right) 10 p^{1} D$ & $-9.541[-2]$ \\
\hline $2 s^{2} 2 p\left({ }^{2} P^{o}\right) 4 f^{1} F$ & $-5.666[-1]$ & $2 s^{2} 2 p\left({ }^{2} P^{o}\right) 7 d^{3} F^{o}$ & $-1.911[-1]$ & $2 s^{2} 2 p\left({ }^{2} P^{o}\right) 9 p{ }^{1} S$ & $-9.417[-2]$ \\
\hline $2 s^{2} 2 p\left({ }^{2} P^{0}\right) 4 f^{3} F$ & $-5.664[-1]$ & $2 s^{2} 2 p\left({ }^{2} P^{o}\right) 7 d^{3} D^{o}$ & $-1.880[-1]$ & $2 s^{2} 2 p\left({ }^{2} P^{o}\right) 10 d^{3} F^{o}$ & $-9.242[-2]$ \\
\hline $2 s 2 p^{2}\left({ }^{4} P\right) 3 p^{3} p^{0}$ & $-5.641[-1]$ & $2 s^{2} 2 p\left({ }^{2} P^{o}\right) 7 d^{3} P^{\circ}$ & $-1.877[-1]$ & $2 s^{2} 2 p\left({ }^{2} P^{o}\right) 10 p p^{3} P$ & $-9.213[-2]$ \\
\hline $2 s^{2} 2 p\left({ }^{2} P^{0}\right) 4 f^{3} G$ & $-5.639[-1]$ & $2 s^{2} 2 p\left({ }^{2} P^{o}\right) 7 d^{1} F^{o}$ & $-1.860[-1]$ & $2 s^{2} 2 p\left({ }^{2} P^{o}\right) 10 d^{1} D^{o}$ & $-9.188[-2]$ \\
\hline $2 s^{2} 2 p\left({ }^{2} P^{0}\right) 4 d^{1} P^{0}$ & $-5.637[-1]$ & $2 s^{2} 2 p\left({ }^{2} P^{\circ}\right) 7 f^{1} F$ & $-1.851[-1]$ & $2 s^{2} 2 p\left({ }^{2} P^{0}\right) 10 d^{3} D^{0}$ & $-9.153[-2]$ \\
\hline $2 s^{2} 2 p\left({ }^{2} P^{o}\right) 4 f^{1} G$ & $-5.628[-1]$ & $2 s^{2} 2 p\left({ }^{2} P^{\circ}\right) 6 f^{3} F$ & $-1.850[-1]$ & $2 s^{2} 2 p\left({ }^{2} P^{o}\right) 10 d^{3} P^{o}$ & $-9.140[-2]$ \\
\hline $2 s^{2} 2 p\left({ }^{2} P^{\circ}\right) 4 f^{3} D$ & $-5.628[-1]$ & $2 s^{2} 2 p\left({ }^{2} P^{\circ}\right) 7 d^{1} P^{\circ}$ & $-1.846[-1]$ & $2 s^{2} 2 p\left({ }^{2} P^{0}\right) 10 f^{1} F$ & $-9.058[-2]$ \\
\hline $2 s^{2} 2 p\left({ }^{2} P^{\circ}\right) 4 f^{1} D$ & $-5.622[-1]$ & $2 s^{2} 2 p\left({ }^{2} P^{\circ}\right) 7 f^{3} G$ & $-1.845[-1]$ & $2 s^{2} 2 p\left({ }^{2} P^{\circ}\right) 9 f^{3} F$ & $-9.054[-2]$ \\
\hline $2 s^{2} 2 p\left({ }^{2} p^{0}\right) 5 s^{3} P^{0}$ & $-4.690[-1]$ & $2 s^{2} 2 p\left({ }^{2} P^{0}\right) 7 f^{3} D$ & $-1.840[-1]$ & $2 s^{2} 2 p\left({ }^{2} P^{o}\right) 10 f^{3} G$ & $-9.038[-2]$ \\
\hline $2 s^{2} 2 p\left({ }^{2} P^{0}\right) 5 s^{1} P^{o}$ & $-4.618[-1]$ & $2 s^{2} 2 p\left({ }^{2} P^{0}\right) 6 f^{1} G$ & $-1.839[-1]$ & $2 s^{2} 2 p\left({ }^{2} P^{o}\right) 10 f^{3} D$ & $-9.015[-2]$ \\
\hline $2 s^{2} 2 p\left({ }^{2} p^{0}\right) 5 p^{3} D$ & $-4.398[-1]$ & $2 s^{2} 2 p\left({ }^{2} P^{o}\right) 7 f^{1} D$ & $-1.838[-1]$ & $2 s^{2} 2 p\left({ }^{2} P^{0}\right) 9 f^{1} G$ & $-9.012[-2]$ \\
\hline $2 s 2 p^{2}\left({ }^{4} P\right) 3 d^{5} F$ & $-4.353[-1]$ & $2 s^{2} 2 p\left({ }^{2} P^{o}\right) 8 s^{3} P^{o}$ & $-1.663[-1]$ & $2 s^{2} 2 p\left({ }^{2} p^{0}\right) 10 d^{1} p^{\circ}$ & $-9.011[-2]$ \\
\hline $2 s^{2} 2 p\left({ }^{2} P^{o}\right) 5 p^{1} P$ & $-4.312[-1]$ & $2 s^{2} 2 p\left({ }^{2} P^{o}\right) 8 s^{1} P^{o}$ & $-1.639[-1]$ & $2 s^{2} 2 p\left({ }^{2} P^{o}\right) 10 d^{1} F^{o}$ & $-9.007[-2]$ \\
\hline $2 s^{2} 2 p\left({ }^{2} p^{0}\right) 5 p{ }^{3} S$ & $-4.233[-1]$ & $2 s 2 p^{2}\left({ }^{2} D\right) 3 p^{3} F^{\circ}$ & $-1.621[-1]$ & $2 s^{2} 2 p\left({ }^{2} p^{0}\right) 10 f^{1} D$ & $-9.006[-2]$ \\
\hline $2 s^{2} 2 p\left({ }^{2} P^{0}\right) 5 p^{3} P$ & $-4.134[-1]$ & $2 s^{2} 2 p\left({ }^{2} P^{o}\right) 8 p{ }^{1} P$ & $-1.574[-1]$ & $2 s 2 p^{2}\left({ }^{4} P\right) 4 p^{3} s^{o}$ & $-5.859[-2]$ \\
\hline $2 s^{2} 2 p\left({ }^{2} p^{0}\right) 5 p p^{1} D$ & $-4.106[-1]$ & $2 s^{2} 2 p\left({ }^{2} P^{0}\right) 8 p{ }^{3} S$ & $-1.561[-1]$ & $2 s 2 p^{2}\left({ }^{4} P\right) 4 p{ }^{5} D^{\circ}$ & $-4.781[-2]$ \\
\hline $2 s 2 p^{2}\left({ }^{4} P\right) 3 d^{5} D$ & $-4.039[-1]$ & $2 s^{2} 2 p\left({ }^{2} P^{\circ}\right) 8 p{ }^{3} D$ & $-1.555[-1]$ & $2 s 2 p^{2}\left({ }^{4} P\right) 4 p^{5} P^{o}$ & $-3.989[-2]$ \\
\hline $2 s 2 p^{2}\left({ }^{4} P\right) 3 d^{5} P$ & $-3.985[-1]$ & $2 s^{2} 2 p\left({ }^{2} P^{o}\right) 8 p{ }^{3} P$ & $-1.549[-1]$ & $2 s 2 p^{2}\left({ }^{4} P\right) 4 p{ }^{5} S^{0}$ & $-1.121[-2]$ \\
\hline $2 s 2 p^{2}\left({ }^{2} D\right) 3 s^{3} D$ & $-3.942[-1]$ & $2 s^{2} 2 p\left({ }^{2} p^{0}\right) 8 p^{1} D$ & $-1.512[-1]$ & & \\
\hline
\end{tabular}

each of the three figures, the cross section given in the lowest panel equals the sum of all the panels above. Figure 1 shows ten panels corresponding to the ground and the ten states included in the CC expansion for the residual ion $\mathrm{C}^{+}$. The lowest panel shows the total cross section in the entire energy range covered by all the states of the residual ion $\mathrm{C}^{+}$. The successively higher panels of Fig. 1 show separately the individual cross sections for photoionization into the $2 s^{2} 2 p\left({ }^{4} P\right)$ state (the second ionization threshold), the ${ }^{2} D$ state (third ionization threshold), and so on up to the $2 p^{3}\left({ }^{2} D^{\circ}\right)$ state of $\mathrm{C}^{+}$. The energy ranges shown for the excited-state photoionization begin at the corresponding ionization threshold and continue up to a little over the highest threshold $2 p^{3}\left({ }^{2} P^{0}\right)$. Note that the threshold energy for each excited state of the residual ion is obtained by adding the ionization energy which is $0.8452 \mathrm{Ry}$ for $\mathrm{C}^{0}$. It can be noticed that all these partial cross sections show a large number of autoionization resonances. Above the ${ }^{2} P^{0}$ state the photoionization cross section is not coupled to any higher states and should be featureless without resonances except for a smooth, monotonically decreasing variation with energy.

Figures 2 and 3 show similar results for the $\mathrm{N}^{+}$and $\mathrm{O}^{2+}$ for the eight states included in the CC expansion for the residual ions $\mathrm{N}^{2+}$ and $\mathrm{O}^{3+}$. It may be seen from Figs. 1-3 that the partial cross sections corresponding to the excited ionization thresholds are considerably smaller than the photoionization cross section into the ground state of the ion, the $2 s^{2} 2 p\left({ }^{2} P^{o}\right)$. However, for a highdensity radiation flux the photoionization and the recombination-rate coefficients of these partial cross sections determine the population rate of the excited states of the residual ion.

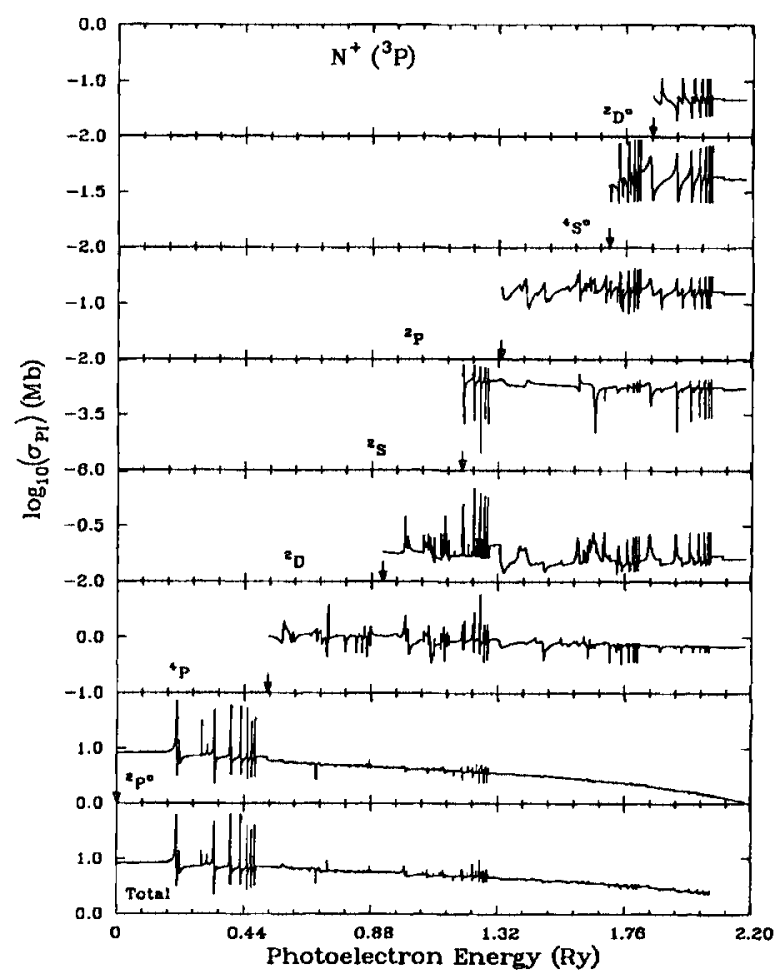

FIG. 2. Photoionization cross sections $\sigma_{\mathrm{Pl}}$, (in $\mathrm{Mb}$ ) for the ground state $2 s^{2} 2 p^{23} P$ of $N^{+}$. The lowest panel shows the total cross sections and the successively higher ones correspond to partial cross section from the ground state leaving the residual ion in the states $2 s^{2} 2 p\left({ }^{2} P^{o}\right), 2 s 2 p^{2}\left({ }^{4} P,{ }^{2} D,{ }^{2} S,{ }^{2} P\right)$, and $2 p^{3}\left({ }^{4} S^{\circ},{ }^{2} D^{\circ}\right)$. The lower $y$-axis limit in each panel corresponds to the partial cross sections indicated, but the upper limit is not shown except for the top one. 
Detailed calculations on the photoionization cross sections from ground state of $\mathrm{C}^{0}$ were carried out by Burke and Taylor [15] and Hofmann and Trefftz [16] using $8 \mathrm{CC}$ and $5 \mathrm{CC}$ target-state expansions, respectively; they used the line-shape formula of Fano to obtain energies and autoionization widths for a few resonances. The present results with 10CC target state expansion of $\mathrm{C}^{0}$ are in good agreement both with the background as well as the resonance positions of the earlier results [15,16]. Additionally the autoionization resonances are more resolved in the present work and show a greater number of resonances belonging to higher excited target states. Henry [17] used close-coupling approximation to calculate the ground-state photoionization cross sections at the threshold and a few other energies for atoms and ions of carbon, nitrogen, and oxygen and used the Seaton fitting formula to obtain the background cross sections. No resonances were considered. The threshold cross sections of Henry for $\mathrm{N}^{+}$and $\mathrm{O}^{2+}$ are lower than the present values, while for $\mathrm{C}^{0}$ it is comparable. The present results of $\mathrm{O}^{2+}$ are a slight improvement over the earlier work of Luo et al. $[18]$ in that the experimental target energies are used along with a finer energy mesh for better resolution of the autoionization resonances.

The Gailities jump. If we consider the photoionization from the ground state to the ground state of the residual ion, i.e.,

$$
2 s^{2} 2 p^{2}\left({ }^{3} P\right)+h v \rightarrow 2 s 2 p\left({ }^{2} P^{o}\right)+e(\epsilon l),
$$

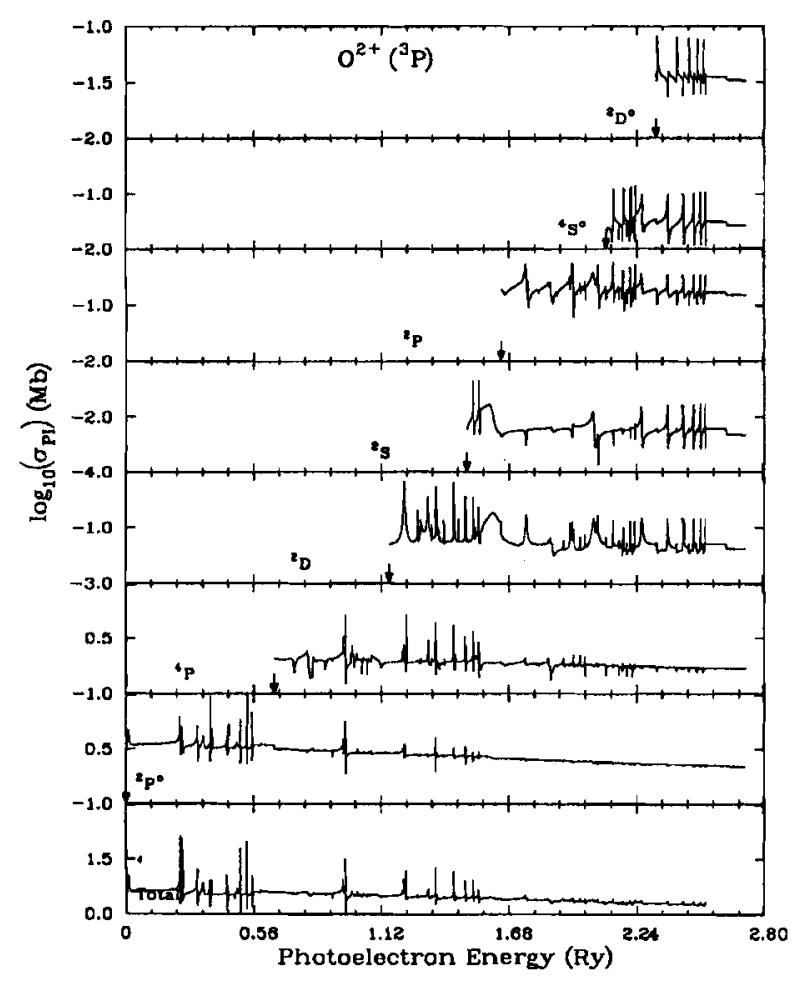

FIG. 3. Photoionization cross sections $\sigma_{\mathrm{PI}}$ (in $\mathrm{Mb}$ ) for the ground state $2 s^{2} 2 p^{23} P$ of $\mathrm{O}^{2+}$ (as in Fig. 2 for $\mathrm{N}^{+}$). then the partial cross sections for (5) exhibit sudden and sharp downward "jumps" as each successively higher ionization threshold is crossed. These are known as Gailities jumps and the phenomenon has been discussed within the context of quantum-defect theory of photoionization by Seaton [14] and Pradhan [19]. Essentially, it involves the loss of flux into the photoionization channels that become open with each excited threshold. Below the threshold the corresponding channels are closed and give rise to Rydberg series of autoionizing resonances converging on to the threshold in question. Once the channels open up at the threshold, the electron flux trapped in the resonances below now goes into the photoelectrons that are free, leaving the residual ion into the higher excited state.

It follows that the magnitude of the Gailities jump in the photoionization process (5) at a given threshold, must be precisely equal to the partial cross section for photoionization into the excited state. Thus the cross section for the photoionization process

$$
2 s^{2} 2 p^{2}\left({ }^{3} P\right)+h v \rightarrow 2 s 2 p^{2}\left({ }^{4} P\right)+e\left(\epsilon^{\prime} l\right),
$$

is equal to the Gailities jump in (5) at the ${ }^{4} P$ threshold.

The process gets more and more complicated as higher thresholds are crossed since at each new threshold the partial cross sections must equal the combined total of the Gailities jumps in each of the other partial cross sections. For example, at the $2 s 2 p^{2}\left({ }^{2} D\right)$ threshold, the cross section for

$$
2 s^{2} 2 p^{2}\left({ }^{3} P\right)+h v \rightarrow 2 s 2 p^{2}\left({ }^{2} D\right)+e\left(\epsilon^{\prime \prime} l\right)
$$

equals the sum of the Gailities jumps in the photoionization cross sections for (5) and (6), since both the cross sections contain resonances of the type ${ }^{2} D n l$ that correspond to the ${ }^{2} D \epsilon l \quad(\epsilon>0)$ channels which open up at ${ }^{2} D$; hence the cross section for both (5) and (6) must be reduced by the corresponding amount.

In Fig. 4 we illustrate the phenomenon of the Gailities jump in the partial cross section for processes (5) and (6) in $\mathrm{C}^{0}$. The vertical drop in the cross section (pointed to by an arrow) in the lower panel of Fig. 4 for $\sigma\left({ }^{3} P \rightarrow{ }^{2} P^{\circ}\right)$ at the ${ }^{4} P$ threshold shows the jump that corresponds to the cross section for process (6). Similarly, the upper panel shows that jump at the ${ }^{2} D$ threshold that corresponds to process (7), but is not equal to the total cross section for (7) [the contribution from (6) and (7) must be added]. It can be noted that while the Gailities average shows a smooth average below the ${ }^{4} P$ threshold (lower panel), it shows a broad resonance below the ${ }^{2} D$ threshold (upper panel). This resonance below the ${ }^{2} D$ threshold belongs to the resonance series of a higher target excited state which is not averaged over because the Gailities average is carried out only for those resonances that converge to the next target excited state. Hence we make a distinction between "weakly" closed channels in the region just below a threshold, which are about to become open, and "strongly" closed channels that belong to higher states and remain closed when the threshold is crossed. 


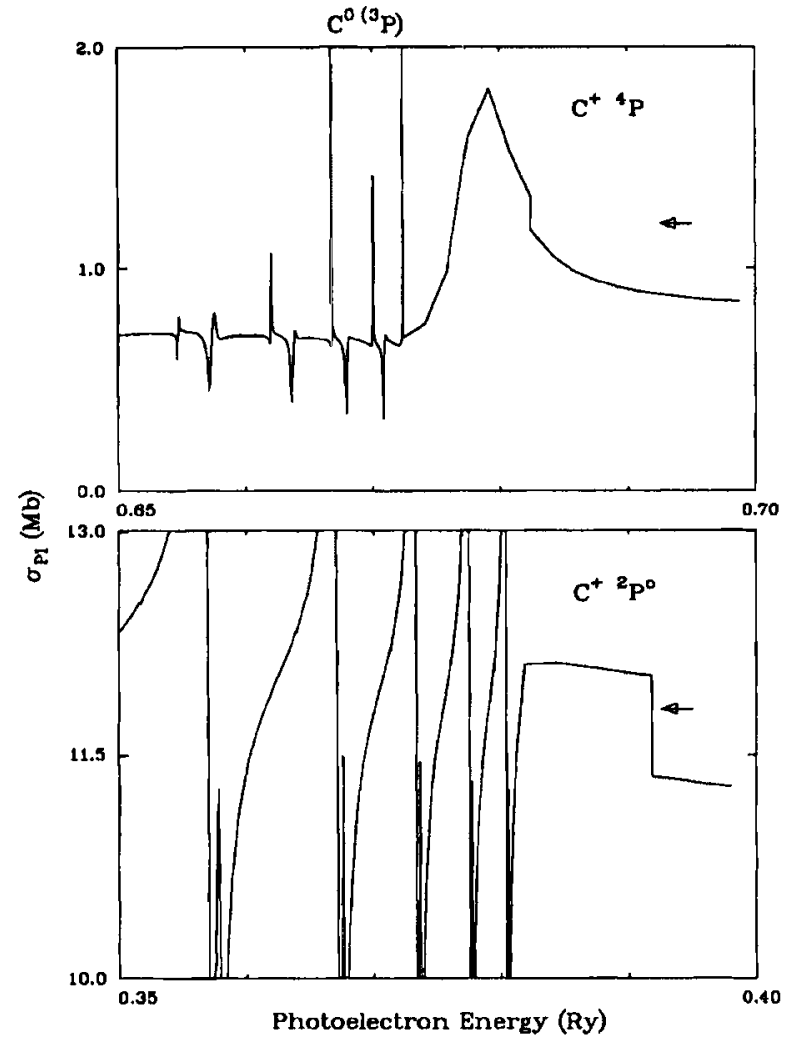

FIG. 4. Illustration of the Gailities jump at the excited target thresholds ${ }^{4} P$ (in the lower panel) and the ${ }^{2} D$ (in the upper panel) in the partial photoionization cross sections of ${ }^{3} P$ ground state of $C^{0}$ leaving the target in the ground state ${ }^{2} P^{o}$ and the excited ${ }^{4} P$ state.

\section{Excited states}

One can easily infer the richness of detail in photoionization cross sections obtained with the methods of the Opacity Project as one considers not only the groundstate photoionization, but the photoionization of the large number of excited states, for example, 158 states of $\mathrm{C}^{0}, 170$ states of $\mathrm{N}^{+}$, and 193 states of $\mathrm{O}^{2+}$ as listed in Table II. It is possible to carry out the calculations for the individual partial cross sections of excited states of atom and ions and these may be of practical importance, for metastable states in particular.

The excited-state photoionization cross sections, although in general smaller than that of the ground state, may, however, be significantly enhanced due to indirect photoionization through autoionizing resonances. Figure 5 shows the photoionization of $2 s 2 p^{3} D^{0}$ excited state of $\mathrm{N}^{+}$where the top part shows the total range of energies encompassing all the excited target states and the lower part illustrates the autoionizing resonances of the $\left({ }^{4} P\right) n l$ series and the $\left({ }^{2} D\right) 3 s$ state below the first threshold ${ }^{4} P$. This is an example of a case that has a very low background cross section but which is effectively large since the resonances are broad and high. The direct photoion-
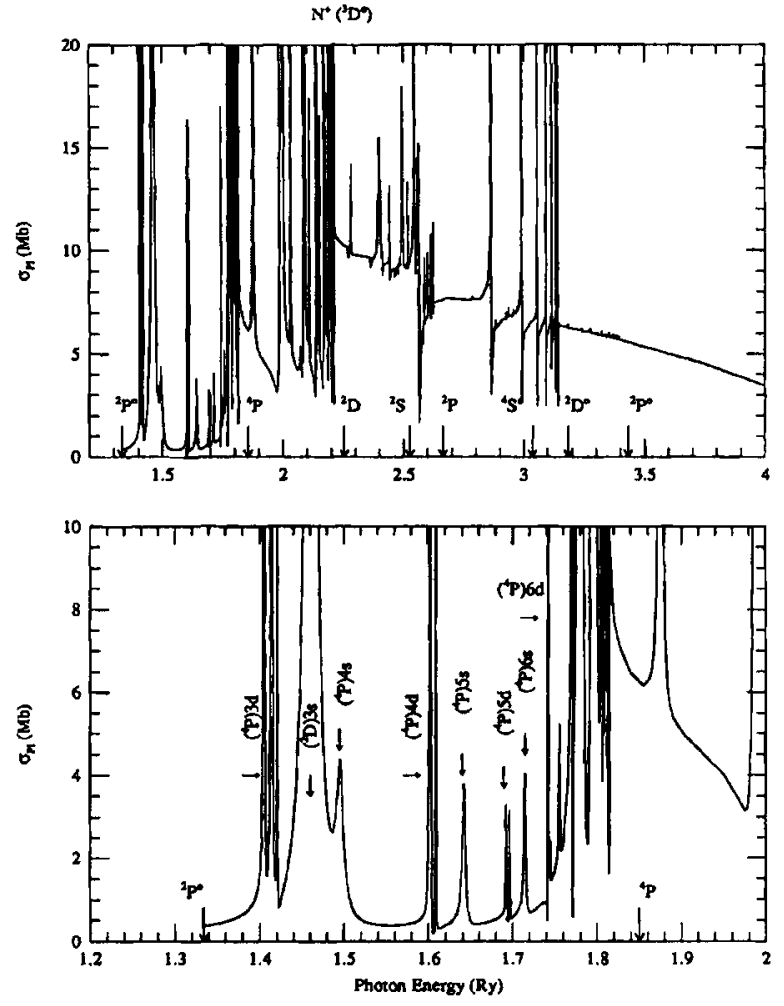

FIG. 5. Photoionization of $2 s 2 p^{33} D^{0}$ excited state of $\mathrm{N}^{+}$; the top part shows the entire range of target-state energies and the lower part illustrates the autoionizing resonances of the $\left({ }^{4} P\right) n l$ series and the $\left({ }^{2} D\right) 3 s$ resonance that lies below the first threshold ${ }^{4} P$.

ization from $2 s 2 p^{33} D^{o}$ to ground state $2 s^{22} P^{o}$ is parentage forbidden. This parentage forbidden photoionization is, however, obtained in the $\mathrm{CC}$ approximation by the admixture of configurations $2 s^{2} 2 p$ and $2 s 2 p^{2}$ of the residual ion. Thus the photoionization of the $2 s 2 p^{3}$ state proceeds mainly through autoionizing resonances of the type $2 s 2 p^{2} n l$, in the region below the first $2 s 2 p^{2}$ state, ${ }^{4} P$. It should be noted that a simpler approximation such as the central field model or the single configuration Hartree-Fock would yield zero cross section in the energy range ${ }^{2} P^{0} \leq E \leq{ }^{4} P$. However, as one can see the effective photoionization cross section in this range is very large due to the indirect process of autoionization. The bottom figure shows that the $\left({ }^{4} P\right)$ ns resonances are broader and are single resonances belonging to the ${ }^{3} P$ state, whereas the $\left({ }^{4} P\right)$ nd resonances are narrower and are multiple resonances belonging to the ${ }^{3} P,{ }^{3} D$, and ${ }^{3} F$ states (the "triplet" structure is delineated in the first set of resonances, ${ }^{4} P 3 d$ ).

In addition to the Rydberg series of resonances converging on to different thresholds of the residual ion, there is an important class of resonances in excited state photoionization cross sections as discussed below.

The photoexcitation of core (PEC) resonances. First 
discussed by $\mathrm{Yu}$ and Seaton [20], the autoionizing resonances that correspond to photoexcitation of core of the residual ion are ubiquitous in the cross sections for photoionization of bound states of a Rydberg series, where the outer electron may considered to be a "spectator" (precisely analogous to the inverse dielectronic recombination process). We consider the transition

$X^{q}\left(S_{i} L_{i} n l\right)+h v \rightarrow X^{g * *}\left(S_{i} L_{i}^{\prime} n l\right) \rightarrow X^{q+1}\left(S_{i} L_{i}\right)+e\left(\epsilon l^{\prime}\right)$, where $h v=E(I P)+\epsilon$ and $S_{i} L_{i} \rightarrow S_{i} L_{i}^{\prime}$ is a dipole-allowed transition in the core ion (in particular the resonance transitions). At photon energies nearly equal to the energy difference $\Delta\left[E\left(S_{i} L_{i}\right)-E\left(S_{i} L_{i}^{\prime}\right)\right]$, the outer Rydberg electron $n l$ plays a less active role than the photoexcitation of the core itself and is usually a highly probable process due to the associated dipole moment. The autoionizing state $X^{q * *}\left(S_{i} L_{i}^{\prime} n l\right)$ decays leaving the residual ion in the initial state (usually the ground state) and a free electron with energy $\epsilon$. Resonances of this type in the photoionization cross sections have been referred to as PEC resonances. It might be noted that if the arrows are reversed, then the equation describes the dielectronicrecombination process.

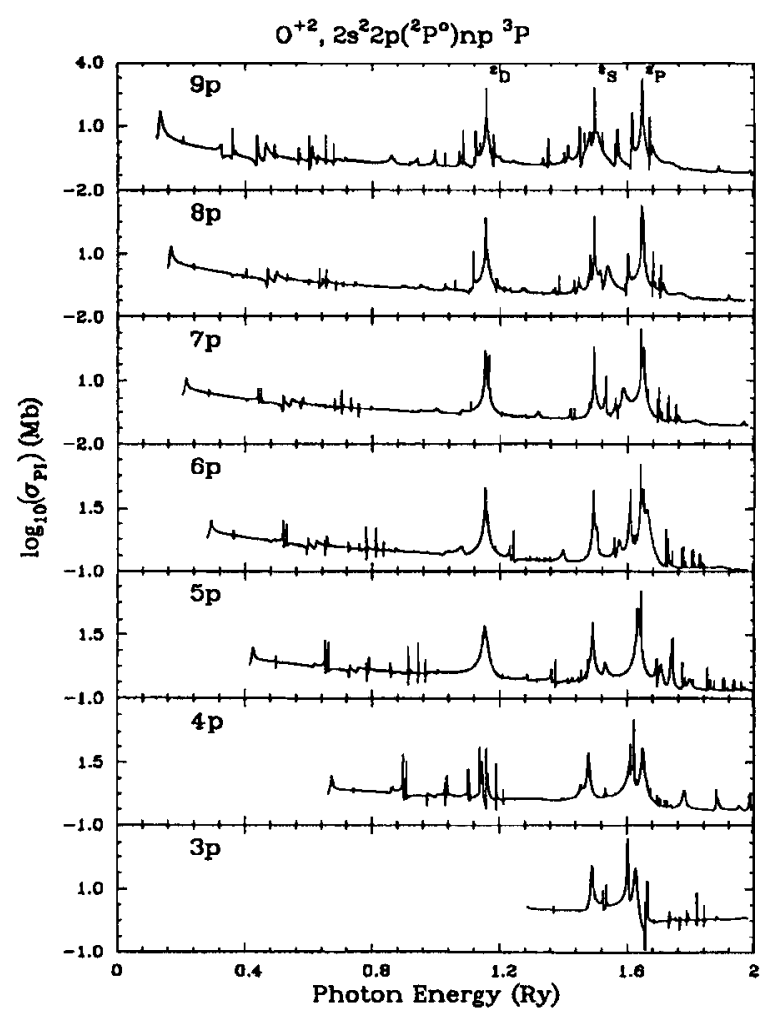

FIG. 6. The photoexcitation-of-core $P E C$ resonances arising from the core transitions of $2 s^{2} 2 p\left({ }^{2} P^{0}\right) \rightarrow 2 s 2 p^{2}\left({ }^{2} D,{ }^{2} S,{ }^{2} P\right)$ states in the photoionization of the bound ${ }^{3} P$ state of the Ryd. berg series $2 s^{2} 2 p\left({ }^{2} P^{o}\right) n p$ of $\mathrm{O}^{2+}$, where $n p$ ranges from $3 p$ to $9 p$. Except for the top one, the upper $y$-axis limit of the panels is not shown.
In general there may be several dipole-allowed core transitions and hence several PEC features, as illustrated in Fig. 6. The figure shows the photoionization of the bound ${ }^{3} P$ states of the Rydberg series $2 s^{2} 2 p\left({ }^{2} P^{o}\right) n p$ of $\mathrm{O}^{2+}$, with $3 \leq n \leq 9$. There are three core dipole transitions within the first two configurations $2 s^{2} 2 p$ and $2 s 2 p^{2}$ of the ground $n=2$ complex:

$$
2 s^{2} 2 p\left({ }^{2} P^{\circ}\right) \rightarrow 2 s 2 p^{2}\left({ }^{2} D,{ }^{2} S,{ }^{2} P\right) .
$$

PEC resonances corresponding to the allowed core transitions can be observed in Fig. 6. The first point of each panel corresponds to the threshold energy for photoionization to the core ground state ${ }^{2} P^{0}$ and it decreases with each successively higher member of the Rydberg series. Hence as one moves up with the Rydberg series, the photoionization energy gets lower, but the PEC resonances remain in the same positions for all members due to the fact that it is the core that is going through the transition while the outer electron remains as a spectator. It can be noted that the first PEC resonances are not seen in the lowest panel since the ionization threshold for $2 s^{2} 2 p\left({ }^{2} P^{o}\right) 3 p{ }^{3} P$, which is $1.288 \mathrm{Ry}$, lies above the first autoionizing energy $1.1545 \mathrm{Ry}$ of the ${ }^{2} P^{\circ}$ to ${ }^{2} D$ transition. The figure shows that the width of the PEC resonances gets narrower, as expected from the $1 / n^{3}$ dependence, along the Rydberg series. This can also be concluded from the uncertainty principle that as the energy of the higher member decreases, the lifetime of the state increases giving a narrower width. The weaker resonances below the PEC's in Fig. 6 are of the ${ }^{4} \mathrm{Pnl}$ type.

\section{ELECTRON-ION RECOMBINATION}

As mentioned earlier one may treat the recombination process as two separate ones: radiative and dielectronic recombination (RR and DR). However, now that one is able to carry out photoionization calculations including the Rydberg series of resonances and their detailed autoionization profiles, one should be able to obtain the total effective recombination cross sections from the principle of detailed balance in the energy region where the resonances are explicitly delineated, i.e., the low-energy region.

The Milne relation, based on detailed balance, may be used to integrate the photoionization cross sections over a Maxwellian distribution of photoelectron energies to obtain recombination rate coefficients (Ref. [6]). However, the problem arises if the resonances in the near threshold region are extremely narrow and yet have autoionization rates larger than the radiative rate.

If we consider the autoionization and radiative processes independently, then following Seaton and Storey [21] we obtain for the dielectronic-recombination-rate coefficient

$\alpha_{d}=\frac{\omega^{* *}}{2 \omega_{+}}\left[\frac{h^{2}}{2 \pi m k T}\right]^{3 / 2} \exp \left[-\frac{E\left(X^{*}\right)}{k T}\right]\left[\frac{A_{a} A_{r}}{A_{a}+A_{r}}\right]$,

where $E\left(X^{* *}\right)=\Delta\left[E\left(X^{* *}\right)-I P(X)\right]$ is the energy of the autoionizing state $X^{* *}$ relative to the residual ion $X^{+}$, 
and $A_{a}$ and $A_{r}$ are the autoionization and radiative probabilities per unit time.

If $A_{a} \gg A_{r}$, then Eq. (8) reduces to

$$
\alpha_{d}=\frac{\omega^{* *}}{2 \omega_{+}}\left(\frac{h^{2}}{2 \pi m k T}\right)^{3 / 2} \exp \left(-\frac{E\left(X^{* *}\right)}{k T}\right) A_{r} .
$$

Equation (9) is the basis of extensive work carried out by Nussbaumer and Storey [10] on low-energy DR. It is not difficult to see that there could be a number of nearthreshold resonances that would contribute to (8); however, since $A_{a}$ is usually orders of magnitude greater than $A_{r}$, the resonances may be very narrow (with relatively small energy widths), but still dominate radiative decay. For example, in atomic units an autoionization probability of $10^{12} \mathrm{sec}^{-1}$ corresponds to only $4.8 \times 10^{-5} \mathrm{Ry}$ in width (or $6.6 \times 10^{-4} \mathrm{eV}$ ). On the scale of broad photoionization features it would be difficult to delineate the profiles of such resonances and a very fine mesh of energies would be needed. As the radiative decay rates are typically $10^{8}-10^{9} \mathrm{sec}^{-1}$, approximation (9) would still apply.

The other factor that makes the near-threshold resonances important in the recombination process is the exponential factor with negative-energy difference relative to the ionization threshold. Nussbaumer and Storey (hereafter referred to as NS) have carried out atomicstructure calculations to predict the positions of these autoionizing states, most of which are not observed in experiments and therefore the energies are not known. NS also estimate the $A_{a}$ from threshold collision strengths for the associated core transitions; however, as discussed above, since $A_{a} \gg A_{r}$, the precise value of $A_{a}$ is not critical to their approximation. While the present $\mathrm{CC}$ calculations should be at least as accurate as the NS work, in terms of the positions of the resonances, they should be a significant improvement in terms of including the resonance profiles, representing the interaction with the background continuum accurately.

\section{A. Recombination-rate coefficients}

We consider the ground-state recombination-rate coefficients calculated according to Eq. (2) at a range of temperatures. The photoionization cross sections for the ground states are calculated in two separate ranges of energies: (i) the near-threshold region, $0 \leq E \leq 0.2 \mathrm{Ry}$, where a fixed energy interval of $0.0001 \mathrm{Ry}$ is employed, and (ii) the energy region from $0.2 \mathrm{Ry}$ up to and above the highest excitation threshold of the residual ion with an effective quantum number mesh and $\Delta v=0.01$, $\nu_{\max }=10$ (as explained in Sec. II C).

In the region of all channels open, i.e., above all target thresholds, the photoionization cross section is expected to be a smooth, monotonically decreasing function of energy. In this region $E>E\left(S_{i} L_{i}\right)_{\max }$, where $S_{i} L_{i}$ is the highest excited state of the residual ion, we take a fixed energy interval of $\Delta E=0.02 \mathrm{Ry}$. Tabulation is continued to energies where the cross sections are expected to have fallen to very low values relative to values at the first ionization threshold. However, it is found that in this re- gion, $E>E\left(S_{i} L_{i}\right)_{\max }$, the cross sections fairly often contain pseudoresonances that must be eliminated in order that accurate rate coefficients may be calculated. Below we discuss the phenomenon in some detail.

\section{Pseudoresonances}

The $(N+1)$-electron bound channel correlation-type functions included in the eigenfunction expansion for the ( $e$ plus ion) system, Eq. (3), usually have eigenvalues that lie in the high-energy region $E>E\left(S_{i} L_{i}\right)_{\max }$. These are expected to be too high to affect near-threshold cross sections and, consequently, rate coefficients at low to moderate temperatures, owing to the exponential cutoff in the Maxwellian. The original intention of the second term in the close-coupling formulation was twofold: (i) to enable the explicit orthogonalization of the continuum orbitals to bound core-ion orbitals of the same symmetry and (ii) to represent short-range correlation, through additional $(N+1)$-electron functions, to augment the correlation with continuum channels in the first sum of the expansion. However, some of the poorly represented $(N+1)$-electron functions, particularly those with pseudoorbitals, give rise to pseudoresonances in the cross sections, which are usually in the higher-energy region of all channels open where approximate methods might be employed to mitigate their effect. The problem has been discussed in earlier works, e.g., in electron-ion scattering [22].

The vast amount of atomic data calculated under the Opacity Project is processed (before mapping it out for opacities calculations) with a computer program (INTFACE) that checks the cross sections and eliminates the region where pseudoresonances are present, replacing it with a fit to the cross sections before pseudoresonances are encountered. In practice it has been difficult to derive an algorithm that would deal with all cases of pseudoresonances. Generally, the fitting in the high-energy region is either (i) $\sigma \sim a \epsilon^{-b}(b>0$ and $\leq 3)$ or (ii) $\sigma \sim\left(\epsilon / \epsilon_{o}\right)^{-3}$; the Kramers fit, in case (i), cannot be obtained. The high-energy extrapolation in case of pseudoresonances implies that in this region some of our cross sections are not of the same accuracy as the cross sections in the lowenergy region encompassing the ionization thresholds included in the $\mathrm{CC}$ expansion.

\section{Results}

Carrying through the integration of the photoionization cross sections according to the Milne relation, Eq. (1), we have calculated recombination-rate coefficients for all states, ground and excited, listed in Table II, that is 159 bound states of $\mathrm{C}^{0}, 171$ of $\mathrm{N}^{+}$, and 194 of $\mathrm{O}^{2+}$. As the present results represent the total, radiative-, and dielectronic-recombination coefficients, we compare these with the earlier calculations of NS for the DR rates for the triplet and singlet symmetries, and with $R R$ rates calculated by us using Henry's [17] threshold photoionization cross sections and the Seaton fitting formula for the ground state and by Gould [23] for the total.

In Table III we present the preliminary results from 
TABLE III. Recombination-rate coefficients in units of $10^{-13} \mathrm{~cm}^{3} \mathrm{sec}^{-1}$ at $10^{4} \mathrm{~K}$. The symbols $g$ and $T$ correspond to ground-state and total rate coefficients, respectively; NS corresponds to Ref. [10]; $t$ corresponds to the sum over all triplets; and $P$ represents values from the present work. For DR values of NS, $T$ represents the sum of the triplet ${ }^{3} P$ and the metastable singlet states ${ }^{1} D$ and ${ }^{1} S$.

\begin{tabular}{|c|c|c|c|c|c|c|c|}
\hline \multirow[t]{2}{*}{ Ion } & \multicolumn{2}{|c|}{$\alpha_{R R}$} & \multicolumn{2}{|c|}{$\alpha_{\mathrm{DR}}(\mathbf{N S})$} & \multicolumn{3}{|c|}{$\alpha_{R}(P)$} \\
\hline & $8^{2}$ & $T^{b}$ & $t$ & $T$ & $g$ & $t$ & $T$ \\
\hline $\mathrm{C}^{0}$ & 1.89 & 4.66 & 0.1 & 1.86 & 2.82 & 3.79 & 6.27 \\
\hline $\mathbf{N}^{+}$ & 6.30 & 22.8 & 19.44 & 19.89 & 8.32 & 19.37 & 26.65 \\
\hline $\mathrm{O}^{2+}$ & 11.65 & 54.3 & 95.43 & 105.05 & 16.19 & 99.42 & 129.81 \\
\hline
\end{tabular}

'Present calculations using photojonization cross sections of Ref. [17] and Seaton's fitting formula.

${ }^{b}$ Reference [23].

our calculations for the ground-state recombination-rate coefficient $\alpha_{R}(g)$; the sum over all bound states of triplet symmetry $\alpha_{R}(t)$ (that eventually cascade down to the ground state as in the NS scheme for DR through the triplet states); and the total sum over the ground and all excited states $\alpha_{R}(T)$, which includes contributions from $n>10$ in the hydrogenic approximation for photoionization cross sections. Comparison of the ground-state and total rate coefficients of the present work indicates a large contribution from the excited states. For example, for $\mathrm{O}^{2+}$, the difference between the ground-state and total rate coefficient indicates that the excited-state contribution is about 7 times larger than that of the ground state. Except for $\mathrm{C}^{0}$, the present rate coefficients for the ground states of $\mathrm{N}^{+}$and $\mathrm{O}^{2+}$ are lower than the sum of the RR values and the DR values of NS (who considered autoionizing states with $n \leq 5$ ). Adding the $\mathrm{RR}$ and $\mathrm{DR}$ contributions, as is usually done in astrophysical applications (Osterbrock [6]), the total rate coefficient is $159.8 \times 10^{-13}$ $\mathrm{cm}^{3} / \mathrm{s}$, which is $23 \%$ higher than the present value of $\alpha_{R}$. This may imply that there are some "missing" resonances in the near threshold region or that some excited bound states of higher angular momenta than that included in our calculations, $L>3$, might contribute significantly. A detailed examination of the autoionizing states included in the atomic-structure calculations of NS does reveal some states that lie just above the first ionization threshold but do not appear in our photoionization calculations with the fine mesh. The other possibility is that the NS approximation may have overestimated the DR contribution; their DR values alone are approximately equal to our total recombination-rate coefficients.

A further, more detailed study is therefore needed to settle the question of these resonances which are clearly important in determining the final value of the total recombination rate coefficient. In Sec. IV B we discuss a method using quantum-defect theory of DR to attempt a solution.

\section{B. Quantum-defect theory of DR}

Following the treatment of Bell and Seaton [9] and the application to radiative damping of autoionizing reso- nances by Pradhan and Seaton [8], we express the generalized scattering matrix as

$$
\bar{\xi}_{e e}=\bar{\chi}_{o o}-\bar{\chi}_{o c}\left[\bar{\chi}_{c c}-g(v) \exp (-2 \pi i v)\right]^{-1} \bar{\chi}_{c o},
$$

where $\bar{\zeta}$ is the electron-electron $(e-e)$ part of the scattering matrix including radiative decay. Considering that the remainder represents the photon-electron $(p-e)$ interaction, unitarity requires that

$$
\bar{\zeta}_{e e}^{\dagger} \bar{\zeta}_{e e}+\xi_{p e}^{\dagger} \bar{\zeta}_{p e}=1
$$

$\xi_{p e}$ is then directly related to the DR probability. The matrix $\bar{\chi}$ is the analytic continuation of the $\bar{S}$ matrix above all thresholds in the region of all channels open (where $\bar{\chi}=\bar{S}$ ). In the region below a given threshold $\bar{\chi}$ may be partitioned into block submatrices $\bar{\chi}_{o o}, \bar{\chi}_{o c}, \bar{\chi}_{c o}$, and $\bar{\chi}_{c c}$ representing open-open, open-closed, closedopen, and closed-closed channel interactions. The factor $g(v)$ is

$$
g(v)=\exp \left(\pi v^{3} A_{r} / z^{2}\right),
$$

where $A_{r}$ is the radiative transition probability.

$\bar{\xi}_{e e}$ may be computed explicitly as a function of $v$, the effective quantum number relative to the threshold of convergence for the closed channels, and hence as a function of energy using Eq. (4). The positions of the poles can then be determined by the condition

$$
\chi_{\mathrm{cc}}-g\left(\nu_{c}\right) \exp \left(-2 \pi i v_{c}\right)=0,
$$

where $\chi_{c c}$ represents the diagonalized matrix $\bar{\chi}_{c c}$.

An approximation made in quantum-defect theory (QDT), as sketched above, is that the closed channels are treated as degenerate, and therefore we have a single effective quantum number variable $\nu_{c}$ for all closed channels. This might result in some error in determining the resonance positions.

An extension of the present work then, necessitated by the problem of "missing" resonances, is to employ the 
QDT and the scattering formulation to determine the autoionizing resonance positions and profiles including radiative decay. This involves significant modification in the computational program STGF that calculates the continuum electron wave functions and hence the $\bar{S}$ matrix.

Another approach would be to carry out an atomicstructure calculation similar to that of NS but with a larger number of configurations to calculate the positions of the resonances more accurately. Both these approaches have drawbacks: the QDT technique may not accurately yield the positions of the low-lying resonances, while the atomic structure calculation does not include the interference between the radiative and autoionization probabilities that are treated independently, as in Eq. (9). However, a combination of the two approaches should yield accurate results and work is in progress along these lines.

\section{CONCLUSION}

Extensive calculations of detailed photoionization cross sections are reported with the additional aim of calculating total electron-ion recombination-rate coefficients. The photoionization cross sections show a wealth of detail not generally considered in the work prior to the Opacity Project, in particular (i) the partial photoionization cross sections for the different excited states of the residual ion, (ii) the dominant autoionization features known as PEC resonances, and (iii) photoionization of a very large number of excited bound states.
It is pointed out that one is able to obtain recombination-rate coefficients, from these photoionization cross sections, for individual states of the atom or the ion as well as the total recombination rate for the ( $e$ plus ion) system in the low-energy region. However, practical problems related to narrow resonances in the near-threshold region must be addressed. A comparison with the Nussbaumer and Storey work based mainly on atomic-structure calculations reveals significant differences, the present results being lower. Attempts are in progress to employ scattering theory formulation and quantum-defect theory to address these discrepancies.

It is planned to extend the present work to other ions in the carbon sequence as well as to other isoelectronic sequences. The present approach should in practice be able to account for both radiative and dielectronic recombination in the low-energy region. However, some effects such as radiation damping of autoionizing resonances $[24,25]$ may become important for highly charged and heavy ions. In addition, certain other considerations related to electron and photon continua may be of importance as inherent, for example, in the unified treatment by Haan and Jacobi [26].

\section{ACKNOWLEDGMENTS}

The present work has been supported by a grant from the National Science Foundation (AST-8996215). S.N.N. also acknowledges support by the College of Mathematical and Physical Sciences of the Ohio State University. The computations were carried out on the Cray Y-MP at the Ohio Supercomputer Center in Columbus, Ohio. We would like to thank Nigel Badnell for helpful comments.
[1] M. J. Seaton, J. Phys. B 20, 6363 (1987).

[2] A. K. Pradhan, Phys. Scr. 35, 840 (1987).

[3] K. A. Berrington, P. G. Burke, K. Butler, M. J. Seaton, P. J. Storey, K. T. Taylor, and Yu Yan, J. Phys. B 20, 6379 (1987).

[4] D. Luo and A. K. Pradhan, J. Phys. B 22, 3377 (1989).

[5] D. Luo and A. K. Pradhan, Phys. Rev. A 41, 165 (1990).

[6] See, for example, D. E. Osterbrock, Astrophysics of Gaseous Nebulae and Active Galactic Nuclei (University Science, Mill Valley, CA, 1989), p. 383.

[7] A. Burgess, Astrophys. J. 141, 1588 (1965).

[8] A. K. Pradhan and M. J. Seaton, J. Phys. B 18, 1631 (1985).

[9] R. H. Bell and M. J. Seaton, J. Phys. B 18, 1589 (1985).

[10] H. Nussbaumer and P. J. Storey, Astron. Astrophys. Suppl. Ser. 56, 293 (1984).

[11] P. G. Burke and W. D. Rob, Adv. At. Mol. Phys. 11, 143 (1975).

[12] A. Hibbert, Comput. Phys. Commun. 9, 141 (1975).

[13] W. Eissner, M. Jones, and H. Nussbaumer, Comput. Phys.
Commun. 8, 270 (1974).

[14] M. J. Seaton, Rep. Prog. Phys. 46, 167 (1983).

[15] P. G. Burke and K. T. Taylor, J. Phys. B 12, 2971 (1979).

[16] H. Hofmann and E. Trefftz, Astron. Astrophys. 82, 256 (1980).

[17] R. J. W. Henry, Astrophys. J. 161, 1153 (1970).

[18] D. Luo, A. K. Pradhan, H. E. Saraph, P. J. Storey, and Yu Yan, J. Phys. B 22, 389 (1989).

[19] A. K. Pradhan, J. Phys. B 11, L729 (1978).

[20] Yu Yan and M. J. Seaton, J. Phys. B 20, 6409 (1987).

[21] M. J. Seaton and P. J. Storey, in Atomic Processes and Applications, edited by P. G. Burke and B. L. Moiseiwitsch (North-Holland, Amsterdam, 1976), p. 134.

[22] R. B. Christensen, D. W. Norcross, and A. K. Pradhan, Phys. Rev. A 34, 4704 (1986).

[23] R. J. Gould, Astrophys. J. 219, 250 (1978).

[24] K. Sakimoto, M. Terao, and K. A. Berrington, Phys. Rev. A 42, 291 (1990).

[25] A. K. Pradhoin, Phys. Rev. Lett. 47, 79 (1981).

[26] S. L. Haan and V. L. Jacobs, Phys. Rev. A 40, 80 (1989). 